\title{
A SPACE-TIME ISOGEOMETRIC METHOD FOR THE PARTIAL DIFFERENTIAL-ALGEBRAIC SYSTEM OF BIOT'S POROELASTICITY MODEL*
}

\author{
JEREMIAS ARF ${ }^{\dagger}$ AND BERND SIMEON ${ }^{\dagger}$
}

\begin{abstract}
Biot's equations of poroelasticity contain a parabolic system for the evolution of the pressure, which is coupled with a quasi-stationary equation for the stress tensor. Thus, it is natural to extend the existing work on isogeometric space-time methods to this more advanced framework of a partial differential-algebraic equation (PDAE) A space-time approach based on finite elements has already been introduced. We present a new weak formulation in space and time that is appropriate for an isogeometric discretization and analyze its convergence properties. Our approach is based on a single variational problem and hence differs from the iterative space-time schemes considered so far. Further, it enables high-order convergence. Numerical experiments that have been carried out confirm the theoretical findings.
\end{abstract}

Key words. Biot's poroelasticity model, isogeometric analysis, space-time discretization, high-order convergence

AMS subject classifications. 76S05, 74F10, 65M12, 65M22, 65M60, 65D07

1. Introduction. Poroelasticity describes the coupling of mechanical deformation with the flow of a fluid in a porous medium, which has numerous applications in engineering. In the quasi-static case, which is most widely adopted, the model takes the form of a partial differential-algebraic equation (PDAE) that calls for appropriate discretizations in space and time. The conventional approach so far relies on the method of lines, starting with a semidiscretization by finite elements in space followed by a time integration of the resulting differential-algebraic system. We here introduce a novel scheme that treats space and time simultaneously and that applies isogeometric analysis (IGA) as discretization. In this way, we extend the framework of space-time methods to PDAE problems and show how the powerful algorithmic machinery of IGA with its spline-based function spaces can be applied to such coupled models.

For more background on poroelasticity, we refer to the example of reservoir engineering, where oil and gas reservoirs as well as more sustainable energy resources like geothermal reservoirs are the subject of research [2, 3, 42]. This includes the problem of induced seismicity caused by the injection or extraction of fluids in the subsurface of the earth, leading to anthropogenic earthquakes [25, 37]. Further references on poroelasticity in earthquake engineering are [15, 17, 41]. The works of Karl von Terzaghi [36] and Maurice Anthony Biot $[10,11,12]$ laid the foundation for poroelasticity and were triggered by the observation of consolidation, which means the volume decrease of a fluid-saturated soil caused by an applied loading and fluid discharge. Today, biophysics and biomedicine also make use of poroelastic models; see, e.g., [28] for the mechanical modelling of living tissues and [38] for a model of the fluid-structure interaction in the human brain.

Our article concentrates on the quasi-static Biot system where the deformation is assumed to be much slower than the fluid flow rate. The corresponding coupled model and its numerical solution has been studied before. Without aiming at completeness, we mention the finite element methods (FEM) for the Biot equations introduced in [30], where Lagrange, TaylorHood, and MINI elements are applied. The authors in [32,33] favor a method involving Raviart-Thomas elements. Further finite element approaches can be found in $[9,27,40]$ and

*Received March 5, 2021. Accepted December 22, 2021. Published online on January 26, 2022. Recommended by Ulrich Langer.

${ }^{\dagger}$ TU Kaiserslautern, Dept. of Mathematics, Gottlieb-Daimler-Str. 48, 67663 Kaiserslautern, Germany ( $\{$ arf, simeon\}@mathematik. uni-kl.de). 
in the dissertations $[8,31]$. Note that there are different versions of the Biot model in the sense that the underlying set of primary unknown variables might differ. For example, in [22] the Biot two-field system, in [27] a Biot three-field, and in [40] a four-field formulation are used. The two main reasons for the varying systems are, on the one hand, the possibility to improve stability properties of the numerical method. On the other hand, sometimes specific variables like the fluid flux are of exceptional interest, and a formulation in which such variables are incorporated is preferred. But three- or four-field formulations suffer from the drawback of an increased number of degrees of freedom.

A different class of methods are the ones based on IGA. In IGA, geometric approximations are avoided, and at the same time the underlying spline spaces offer various properties, including increased global smoothness. Isogeometric methods for the Biot system are considered in $[7,24]$. An iterative method for solving Biot's model numerically based on a finite element discretization is introduced in [4]. In the latter reference, the authors analyze a space-time scheme, i.e., the time variable itself is discretized with continuous or discontinuous finite elements.

In this paper we also follow the idea of space-time discretizations and use the approach of [26] for a parabolic evolution equation as the starting point. The extension to the Biot two-field system is not straightforward since we are facing here several challenges. Firstly, an appropriate weak formulation for both pressure and displacement variables has to be derived. Secondly, the treatment of the elastic momentum balance requires special care as it represents a constraint with respect to the time axis. Thirdly and finally, the pressure variable is known to be sensitive to oscillations that call for additional measures. A space-time method directly leads to a linear system that includes all time steps and hence is much larger than a spatial discretization alone. However, by a closer inspection of the linear system, one notices a staircase structure that reflects the propagation of the solution over time and that results in a very sparse matrix. With appropriate fast iterative and parallel solvers, the linear system can be tackled as a whole, but in our work, the focus is on the discretization itself. We also point out that in principle the space-time scheme can be employed to introduce full adaptivity in space and time, which comprises in particular local time step changes in combination with local spatial refinement.

The outline of the paper is as follows: In Section 2 the Biot system is explained briefly while in Section 3 we introduce the space-time method and the underlying space-time discretization. Section 4 presents a convergence result, and in the last part we discuss numerical examples.

The notation that we use is fairly standard. We write the scalar Sobolev spaces over an open domain $D$ as $H^{k}(D)$ for some $k \in \mathbb{N}$, with $L^{2}(D)=H^{0}(D)$. The standard scalar product of the Lebesgue space $L^{2}(D)$ is denoted by $\langle\cdot, \cdot\rangle_{L^{2}(D)}$. In case of vector-valued Sobolev spaces we use a bold-type notation, for example, $\boldsymbol{H}^{k}(D):=\left(H^{k}(D), \ldots, H^{k}(D)\right)$, etc. Moreover, $\|\cdot\|_{L^{2}(D)},\|\cdot\|_{L^{2}(D)},\|\cdot\|_{H^{k}(D)},\|\cdot\|_{H^{k}(D)}$ represent the norms induced by the inner products in the respective spaces. Finally, $\nabla_{x}$ denotes the classical nabla operator in the spatial coordinates.

2. The Biot system. In this section we outline the coupled model of Biot, following the references $[3,32,34]$. The porous medium consists of a solid skeleton and permeable voids (pores) that are filled with some fluid. The medium is identified with a bounded Lipschitz domain $\Omega \subset \mathbb{R}^{d}, d \in\{1,2,3\}$. The model variables are the time-dependent mechanical displacement field $\boldsymbol{u}(t): \Omega \rightarrow \mathbb{R}^{d}$ as well as the fluid pore pressure $p(t): \Omega \rightarrow \mathbb{R}$ and the 
fluid flux $\boldsymbol{q}(t): \Omega \rightarrow \mathbb{R}^{d}$, with $t$ as time. Flux and pressure are connected via Darcy's law

$$
\boldsymbol{q}=-\frac{\boldsymbol{K}}{\eta_{f}}\left(\nabla_{x} p-\rho_{f} \boldsymbol{g}\right)
$$

which gives a linear relation between the pressure gradient and the flux. In the last equation, $\boldsymbol{K}$ denotes the permeability tensor represented by a spd (symmetric positive-definite) matrix, $\eta_{f}$ denotes the fluid viscosity, $\rho_{f}$ the fluid density, and $\boldsymbol{g}$ some body force. For simplicity, we ignore the body force in the sequel.

The first governing equation of the Biot system can be derived by means of the balance of momentum and linear elasticity, namely

$$
-\nabla_{x} \cdot \tilde{\boldsymbol{\sigma}}(\boldsymbol{u}, p)=\boldsymbol{f},
$$

where $f$ is a given force distribution. In this equation, $\tilde{\boldsymbol{\sigma}}=\boldsymbol{\sigma}-b p \boldsymbol{I}$ denotes the total stress tensor, and $b$ is the so-called Biot-Willis coefficient. Assuming a linear-elastic behavior, the solid phase satisfies Hooke's law $\sigma_{i j}=C_{i j k l} \varepsilon_{k l}$ (Einstein's notation) with $C=\left(C_{i j k l}\right)$ being the elasticity tensor, $\boldsymbol{\varepsilon}(\boldsymbol{u})=\left(\nabla_{x} \boldsymbol{u}+\nabla_{x} \boldsymbol{u}^{t}\right) / 2$ the strain tensor, and $\boldsymbol{\sigma}$ the stress tensor. Furthermore, we assume a quasi-static behavior, where second-order time derivatives are neglected. For the special case of an isotropic and homogeneous solid, we can simplify the elasticity tensor to $C_{i j k l}=\lambda\left(\delta_{i j} \delta_{k l}\right)+\mu\left(\delta_{i k} \delta_{j l}+\delta_{i l} \delta_{j k}\right)$, where $\lambda$ and $\mu$ are the Lamé constants and $\delta$ the Kronecker delta.

The second governing equation is obtained from a conservation law for the fluid phase, which reads

$$
\partial_{t}\left(c_{0} p+b \nabla_{x} \cdot \boldsymbol{u}\right)+\nabla_{x} \cdot \boldsymbol{q}=g .
$$

Here, we can interpret $c_{0} p+b \nabla_{x} \cdot \boldsymbol{u}$ as the fluid content and $g$ as a fluid source term. The constant $c_{0}$ is the constrained specific storage coefficient, and in applications it is often close to zero. Both, (2.1) and (2.2) together with Darcy's law lead to the Biot two-field system:

$$
\begin{array}{r}
-\nabla_{x} \cdot \tilde{\boldsymbol{\sigma}}(\boldsymbol{u}, p)=\boldsymbol{f}, \\
\partial_{t}\left(c_{0} p+b \nabla_{x} \cdot \boldsymbol{u}\right)-\nabla_{x} \cdot \frac{\boldsymbol{K}}{\eta_{f}} \nabla_{x} p=g .
\end{array}
$$

As initial conditions we require $p(0)=0$ and $\boldsymbol{u}(0)=\mathbf{0}$, i.e., pressure and displacement are zero at the initial time $t=0$. For the boundary conditions, we introduce two partitions $\overline{\Gamma_{u}} \cup \overline{\Gamma_{t}}=\partial \Omega$ and $\overline{\Gamma_{p}} \cup \overline{\Gamma_{f}}=\partial \Omega$ of the boundary $\partial \Omega$ of the spatial domain with $\Gamma_{u} \cap \Gamma_{t}=\emptyset$ and $\Gamma_{p} \cap \Gamma_{f}=\emptyset$. Then we choose

$$
\begin{array}{rlrlrl}
\tilde{\boldsymbol{\sigma}} \cdot \boldsymbol{n}_{x} & =\boldsymbol{t}_{n} & & \text { on } \Sigma_{t}:=\Gamma_{t} \times(0, T), & & (\text { tension BC) } \\
\mathcal{K} \nabla_{x} p \cdot \boldsymbol{n}_{x} & =v_{f} & & \text { on } \Sigma_{f}:=\Gamma_{f} \times(0, T), & (\text { flux BC }) \\
\boldsymbol{u} & =0 & & \text { on } \Sigma_{u}:=\Gamma_{u} \times(0, T), & \\
p=0 & & \text { on } \Sigma_{p}:=\Gamma_{p} \times(0, T) . &
\end{array}
$$

Here we set $\mathcal{K}:=\eta_{f}^{-1} \boldsymbol{K}$. Moreover, $\boldsymbol{n}_{x}$ denotes the outer unit normal vector. A study of the existence of (weak) solutions to the Biot system can be found in [34]; cf. the following remark.

REMARK 2.1. Showalter's study of the existence and uniqueness of solutions in [34] utilizes the theory of implicit evolution equations and an underlying weak formulation based on equations in the dual spaces $\boldsymbol{V}^{\prime}$ and $V^{\prime}$, where $V:=\left\{v \in H^{1}(\Omega) \mid v=0\right.$ on $\left.\Gamma_{p}\right\}$, 


\section{ETNA}

Kent State University and

Johann Radon Institute (RICAM)

TABLE 2.1

Material parameters.

\begin{tabular}{ccc} 
Parameter & phy. unit & Explanation \\
\hline $\boldsymbol{K}$ & {$\left[\mathrm{m}^{2}\right]$} & permeability tensor \\
$0<\eta_{f}$ & {$\left[\mathrm{~N} \mathrm{~s} / \mathrm{m}^{2}\right]$} & viscosity of the fluid \\
$0<\rho_{f}$ & {$\left[\mathrm{~kg} / \mathrm{m}^{3}\right]$} & density of the fluid \\
$0<b$ & {$[1]$} & Biot-Willis coefficient \\
$0 \leq c_{0}$ & {$\left[\mathrm{~m}^{2} / \mathrm{N}\right]$} & constrained specific storage
\end{tabular}

TABLE 2.2

Model quantities.

\begin{tabular}{ccc} 
Variable & phy. unit & Explanation \\
\hline$\tilde{\boldsymbol{\sigma}}$ & {$\left[N / \mathrm{m}^{2}\right]$} & total stress tensor \\
$\boldsymbol{C}$ & {$\left[N / \mathrm{m}^{2}\right]$} & elasticity tensor \\
$\boldsymbol{\varepsilon}$ & {$[1]$} & lin. strain tensor \\
$\boldsymbol{q}$ & {$[\mathrm{m} / \mathrm{s}]$} & volumetric flux \\
$\boldsymbol{u}$ & {$[\mathrm{m}]$} & displacement \\
$p$ & {$\left[N / m^{2}\right]$} & fluid pressure
\end{tabular}

and $\boldsymbol{V}:=\left\{\boldsymbol{v} \in \boldsymbol{H}^{1}(\Omega) \mid \boldsymbol{v}=0\right.$ on $\left.\Gamma_{u}\right\}$. For a detailed explanation we refer to [34, Section 3]. Showalter considers Neumann-type boundary conditions that differ from those assumed here, and furthermore, he allows for more general initial conditions accompanied by some compatibility condition. Then, in view of [34, Theorem 3.1], one can guarantee a weak solution under relatively mild assumptions. Namely, if all the source and boundary functions are at least of class $C^{\alpha}\left([0, T] ; L^{2}\right)$, i.e., Hölder continuous in time, then one obtains a unique weak solution $p:(0, T] \rightarrow V$ and $\boldsymbol{u}:(0, T] \rightarrow \boldsymbol{V}$ provided that $\int_{\Gamma_{p}} 1 d s>$ 0 . Additionally, Showalter shows the regularity $\boldsymbol{u} \in C^{0}([0, T] ; \boldsymbol{V}) \cap C^{1}((0, T] ; \boldsymbol{V})$ and $\|p(t)\|_{H^{2}(\Omega)},\|\boldsymbol{u}(t)\|_{\boldsymbol{H}^{2}(\Omega)} \leq C / t, 0<t \leq T$, for some constant $C$. Furthermore, for $c_{0}>0$ we have $p \in C^{0}\left([0, T] ; L^{2}(\Omega)\right) \cap C^{1}\left((0, T] ; L^{2}(\Omega)\right)$, and at least in the case of a smoothly bounded domain $\Omega$ together with smooth boundary conditions and source functions, one obtains the smoothness of the pressure $p$ in space and time, even for $c_{0}=0$. Below, for the derivation of the space-time formulation, we consider solutions in space-time Sobolev spaces, and in order to stay on the safe side, we suppose that $\left.p \in H^{2}(\Omega \times(0, T))\right)$ and $\boldsymbol{u} \in \boldsymbol{H}^{3}(\Omega \times(0, T))$. Thus, our assumptions go beyond the theoretical foundations laid by Showalter. Nevertheless, the numerical tests at the end of this article and the fact that the analysis presented here is still valid for less regular functions imply that we get a meaningful method despite the mentioned difference in the boundary conditions and the mentioned regularity issue.

To show the existence of discrete solutions within the scope of our proposed method, we have to postulate the next assumption.

ASSUMPTION 2.2. Let the boundary parts $\Gamma_{u}$ and $\Gamma_{p}$ have positive measure, meaning that $0<\int_{\Gamma_{u}} 1 d s, \int_{\Gamma_{p}} 1 d s$. Besides, let the tensor $\mathcal{K}(\boldsymbol{x})$ be uniformly elliptic and bounded in $\Omega$, and without loss of generality, we assume $c_{0} \leq 1$.

The most important model and material parameters are summarized in the Tables 2.1 and 2.2 below.

3. The space-time discretization and the discrete variational formulation. Regarding the discretization of the Biot system, we first look at the basics of isogeometric analysis and proceed with the derivation of a corresponding discrete space-time variational method.

3.1. Isogeometric analysis. Introduced by Hughes et al. [23], the concept of IGA was developed in the last 15 years into a powerful tool in numerical analysis. The basic idea is the simultaneous use of spline functions for the geometric modelling and the definition of the discrete spaces. IGA is able to represent various complex and curved-boundary domains exactly, and furthermore one has the possibility to easily increase or lower the smoothness of functions in the discrete spaces. Following [5, 6, 14] for a brief exposition, we call for some $r \in \mathbb{N}$ an increasing sequence of real numbers $\Xi:=\left\{\xi_{1} \leq \xi_{2} \leq \cdots \leq \xi_{n+r+1}\right\}$ a knot vector, where we assume that $0=\xi_{1}=\xi_{2}=\cdots=\xi_{r+1}, \xi_{n+1}=\xi_{n+2}=\cdots=\xi_{n+r+1}=1$, and we call such knot vectors $r$-open. Further, the multiplicity of the $j$-th knot is denoted by 


\section{ETNA}

Kent State University and

Johann Radon Institute (RICAM)

$m\left(\xi_{j}\right)$. Then the univariate B-spline functions $\hat{B}_{j, r}(\cdot)$ of degree $r$ corresponding to a given knot vector $\Xi$ is defined recursively by the Cox-DeBoor formula:

$$
\hat{B}_{j, 0}(\zeta):= \begin{cases}1, & \text { if } \zeta \in\left[\xi_{j}, \xi_{j+1}\right), \\ 0, & \text { else }\end{cases}
$$

and if $r \in \mathbb{N}_{\geq 1}$, then we let

$$
\hat{B}_{j, r}(\zeta):=\frac{\zeta-\xi_{j}}{\xi_{j+r}-\xi_{j}} \hat{B}_{j, r-1}(\zeta)+\frac{\xi_{j+r+1}-\zeta}{\xi_{j+r+1}-\xi_{j+1}} \hat{B}_{j+1, r-1}(\zeta),
$$

where one sets $0 / 0=0$ to obtain well-definedness.

The multivariate extension of the last spline definition is achieved by a tensor product construction. In other words, for a given tensor knot vector $\boldsymbol{\Xi}:=\Xi_{1} \times \cdots \times \Xi_{d}$, where the $\Xi_{l}=\left\{\xi_{1}^{l}, \ldots, \xi_{n_{l}+r_{l}+1}^{l}\right\}, l=1, \ldots, d$, are $r_{l}$-open, and a given degree vector $\boldsymbol{r}:=\left(r_{1}, \ldots, r_{d}\right)$, we set for the multivariate case

$$
\hat{B}_{\boldsymbol{i}, \boldsymbol{r}}(\boldsymbol{\zeta}):=\prod_{l=1}^{d} \hat{B}_{i_{l}, r_{l}}\left(\zeta_{l}\right), \quad \boldsymbol{\zeta}:=\left(\zeta_{1}, \ldots, \zeta_{d}\right), \quad \forall \boldsymbol{i} \in \mathbf{I},
$$

with $d$ being the underlying dimension of the parametric domain $\hat{\Omega}=(0,1)^{d}$ and $\mathbf{I}$ the multi-index set $\mathbf{I}:=\left\{\left(i_{1}, \ldots, i_{d}\right) \mid 1 \leq i_{l} \leq n_{l}, l=1, \ldots, d\right\}$. To enlarge the possibilities of the representation of geometric objects, one can generalize the definition of B-splines to rational B-splines. Namely, choosing strictly positive weights $0<w_{\boldsymbol{i}}, \boldsymbol{i} \in \mathbf{I}$, and exploiting the notation from above, we introduce the weight function

$$
W(\boldsymbol{\zeta}):=\sum_{\boldsymbol{i} \in \mathbf{I}} w_{\boldsymbol{i}} \hat{B}_{\boldsymbol{i}, \boldsymbol{r}}(\boldsymbol{\zeta})
$$

We define the non-uniform rational B-spline (NURBS) basis functions $\hat{N}_{i, r}(\zeta)$ with respect to the weight function $W$ as follows:

$$
\hat{N}_{\boldsymbol{i}, \boldsymbol{r}}(\boldsymbol{\zeta}):=w_{\boldsymbol{i}} \hat{B}_{\boldsymbol{i}, \boldsymbol{r}}(\boldsymbol{\zeta})(W(\boldsymbol{\zeta}))^{-1}, \quad \forall \boldsymbol{i} \in \mathbf{I} .
$$

B-splines (and the same holds for NURBS) fulfil several properties, and for our purposes the most important ones are:

- If for all internal knots the multiplicity satisfies $1 \leq m\left(\xi_{j}^{l}\right) \leq m \leq r \leq r_{l}$, for all $l$, then the B-spline basis functions $\hat{B}_{\boldsymbol{i}, \boldsymbol{r}}$ are globally $C^{r-m}$-continuous.

- The B-splines $\left\{\hat{B}_{\boldsymbol{i}, \boldsymbol{r}} \mid \boldsymbol{i} \in \mathbf{I}\right\}$ are linearly independent.

Back to the Biot problem, the aim is the definition of a space-time discretized variational formulation. Consequently, we consider as in [26] the space-time cylinder $\mathcal{Q}=\Omega \times(0, T)$. This so-called physical domain is assumed to be parametrized by means of NURBS or Bsplines, respectively. More precisely, we have a parametrization of the form

$$
\tilde{\mathbf{\Phi}}:(0,1)^{d+1}=: \hat{\mathcal{Q}} \rightarrow \mathcal{Q}, \quad \tilde{\boldsymbol{\zeta}} \mapsto \sum_{\tilde{\boldsymbol{i}} \in \tilde{\mathbf{I}}} \tilde{C}_{\tilde{\boldsymbol{i}}} \hat{N}_{\tilde{\boldsymbol{i}}, \tilde{\boldsymbol{r}}}(\tilde{\boldsymbol{\zeta}})
$$

where the $\tilde{C}_{\tilde{\boldsymbol{i}}}=\left(C_{\boldsymbol{i}}, t_{i_{d+1}}\right) \in \mathbb{R}^{d+1}$ are the control points and

$$
\tilde{\mathbf{I}}=\left\{\left(i_{1}, \ldots, i_{d}, i_{d+1}\right) \in \mathbf{I} \times \mathrm{I}_{t}\right\}, \quad \tilde{\boldsymbol{\zeta}}=\left(\boldsymbol{\zeta}, \zeta_{d+1}\right), \quad \text { and } \quad \tilde{\boldsymbol{r}}=\left(\boldsymbol{r}, r_{d+1}\right),
$$




\section{ETNA}

Kent State University and

Johann Radon Institute (RICAM)

for suitable index sets I and $\mathrm{I}_{t}$. Due to the product structure of the space-time cylinder, we can assume that the parametrization can be written as

$$
\tilde{\mathbf{\Phi}}: \hat{\mathcal{Q}} \rightarrow \Omega \times(0, T), \quad \tilde{\boldsymbol{\zeta}} \mapsto\left(\boldsymbol{\Phi}(\boldsymbol{\zeta}), \Phi\left(\zeta_{d+1}\right)\right),
$$

with

$$
\mathbf{\Phi}(\boldsymbol{\zeta})=\sum_{\boldsymbol{i} \in \mathbf{I}} C_{\boldsymbol{i}} \hat{N}_{\boldsymbol{i}, \boldsymbol{r}}(\boldsymbol{\zeta}) \quad \text { and } \quad \Phi\left(\zeta_{d+1}\right)=\sum_{i \in \mathrm{I}_{t}} t_{i} \hat{B}_{i, r_{d+1}}\left(\zeta_{d+1}\right)=T \zeta_{d+1}
$$

Given such a parametrization, the knots stored in the knot vector $\tilde{\Xi}:=\Xi \times \Xi_{d+1}$ corresponding to the underlying NURBS and splines determine a mesh in the parametric domain $\hat{\mathcal{Q}}$, namely

$\hat{M}:=\left\{K_{j}:=\left(\psi_{j_{1}}^{1}, \psi_{j_{1}+1}^{1}\right) \times \cdots \times\left(\psi_{j_{d+1}}^{d+1}, \psi_{j_{d+1}+1}^{d+1}\right) \mid \boldsymbol{j}=\left(j_{1}, \ldots, j_{d+1}\right)\right.$, with $\left.1 \leq j_{i}<N_{i}\right\}$,

and with

$$
\tilde{\boldsymbol{\Psi}}=\left\{\psi_{1}^{1}, \ldots, \psi_{N_{1}}^{1}\right\} \times \cdots \times\left\{\psi_{1}^{d+1}, \ldots, \psi_{N_{d+1}}^{d+1}\right\}
$$

being the knot vector $\tilde{\Xi}$ without knot repetitions.

The image of this mesh under the mapping $\tilde{\boldsymbol{\Phi}}$, i.e., $\mathcal{M}:=\{\tilde{\boldsymbol{\Phi}}(\hat{K}) \mid \hat{K} \in \hat{M}\}$, gives us a mesh structure in the physical domain. By inserting knots without changing the parametrization, we can refine the mesh, which yields the concept of $h$-refinement [14, 23]. Actually, in the IGA framework, two additional techniques for enlarging the basis function spaces are common. On the one hand there is the p-refinement, meaning the elevation of the underlying polynomial degrees, and on the other hand we have k-refinement, which can be interpreted as a combination of $h$ - and p-refinement. The latter refinement procedure is composed of a degree elevation step followed by the insertion of single knots. This leads to increased smoothness at the new knot, whereas for classical p-refinement, the interelement regularity is kept. Again we refer to [23] for more information on refinement strategies. Here we focus on $h$-refinement in the sense that the polynomial degrees and global regularity of the basis functions are considered to be given, and the approximation estimates below will be stated with respect to the mesh size $h$, which is defined by $h:=\max \left\{h_{\mathcal{K}} \mid \mathcal{K} \in \mathcal{M}\right\}$, where $h_{\mathcal{K}}=\operatorname{diam}(\mathcal{K})$ is the diameter of the mesh element $\mathcal{K}$. In particular, we assume for the $h$-refinement or the mesh refinement, respectively, a preservation of the inner knot multiplicities. For the rest of this article, we assume the mesh to be regular as defined next:

ASSUMPTION 3.1 (Regular mesh). The parametrization mapping is smooth on the closure of each mesh element $\overline{\hat{K}}, \hat{K} \in \hat{M}$, and has a smooth inverse, meaning that $\tilde{\boldsymbol{\Phi}}_{\mid} \in C^{\infty}(\overline{\hat{K}})$, $\tilde{\boldsymbol{\Phi}}_{\mid}^{-1} \in C^{\infty}(\tilde{\boldsymbol{\Phi}}(\overline{\hat{K}}))$. Further, there is a constant $0<c_{M}<\infty$ independent of the mesh refinement such that for the element sizes it holds that $h_{\mathcal{K}} \leq h \leq c_{M} h_{\mathcal{K}}$ for all mesh elements $\mathcal{K} \in \mathcal{M}$. Additionally, for the coarsest mesh, the boundary segments $\Sigma_{u}$ and $\Sigma_{p}$ are the unions of full boundary mesh faces.

Clearly the global mesh is composed of a spatial mesh and a mesh in the time interval $(0, T)$ as consequence of the product structure. Therefore, one can introduce a spatial mesh size $h_{S}$ and a mesh size in the time domain $h_{T}$ in an analogous manner. Although the mesh sizes $h_{S}, h_{T}$ are more convenient for our considerations, we also keep the global mesh size $h$ to shorten the notation.

Lastly, we define the discrete spaces, following the isogeometric paradigm, which are used below for the discretized variational formulation via

$$
\mathcal{V}_{h, \tilde{\boldsymbol{r}}}:=\operatorname{span}\left\{v_{h}=\hat{N}_{\tilde{\boldsymbol{i}}, \tilde{\boldsymbol{r}}} \circ \tilde{\boldsymbol{\Phi}}^{-1} \mid \tilde{\boldsymbol{i}} \in \tilde{\mathbf{I}}\right\},
$$






FIG. 3.1. We exploit the tensor product structure of $\mathcal{Q}$ for parametrizing the space-time cylinder.

spanned by the push-forwards of the NURBS basis functions. Although we stated the test spaces using NURBS, many geometries can be represented accurately already by means of B-splines, and in this case we obtain a constant weight function $W$. This can reduce the computational effort in applications significantly, and indeed in the IGA context, B-splines are often the preferred basis functions. Nevertheless, in this article we allow for NURBS push-forwards as well.

For simplicity we assume the same polynomial degree in each spatial coordinate direction and write $r_{T}$ for the polynomial degree with respect to the time parameter. Based on this we can define the test spaces for the pressure $p$ and the displacement $\boldsymbol{u}$. Let $r_{S} \in \mathbb{N}_{\geq 1}$. We set

$$
\mathcal{V}_{h, r_{S}, r_{T}}:=\mathcal{V}_{h, \tilde{\boldsymbol{r}}} \quad \text { with } \quad \tilde{\boldsymbol{r}}=\left(r_{S}, \ldots, r_{S}, r_{T}\right) .
$$

Let $r_{u}$ and $r_{p}$ denote the underlying spatial polynomial degrees for the displacement and the pressure. Then the discrete displacement and pressure spaces are

$$
\begin{aligned}
\mathcal{V}_{h, r_{u}, r_{T}} & :=\left(\mathcal{V}_{h, r_{u}, r_{T}}\right)^{d} \cap\left\{\boldsymbol{v} \in\left(C^{0}(\mathcal{Q})\right)^{d} \mid \boldsymbol{v}=0 \text { on } \Sigma_{u} \cup \Sigma_{0}\right\}, \\
\mathcal{W}_{h, r_{p}, r_{T}} & :=\mathcal{V}_{h, r_{p}, r_{T}} \cap\left\{q \in C^{0}(\mathcal{Q}) \mid q=0 \text { on } \Sigma_{p} \cup \Sigma_{0}\right\},
\end{aligned}
$$

with $\Sigma_{0}:=\Omega \times\{0\}$. We remark that it is possible to write these function spaces as product spaces, namely

$$
\mathcal{V}_{h, r_{u}, r_{T}}=V_{h_{S}, r_{u}} \otimes V_{h_{T}, r_{T}} \quad \text { and } \quad \mathcal{W}_{h, r_{p}, r_{T}}=W_{h_{S}, r_{p}} \otimes V_{h_{T}, r_{T}},
$$

where $\boldsymbol{V}_{h_{S}, r_{u}}, W_{h_{S}, r_{p}}$ are NURBS-based approximation spaces corresponding to the spatial discretization and $V_{h_{T}, r_{T}}$ is the finite-dimensional space for the time discretization.

3.2. The discrete space-time variational formulation. She starting point for the discretized variational formulation is the classical Biot two-field model. For the derivation we consider the solution to satisfy $p \in H^{2}(\mathcal{Q})$ and $\boldsymbol{u} \in \boldsymbol{H}^{3}(\mathcal{Q})$ while the right-hand sides fulfil $\boldsymbol{f} \in \boldsymbol{H}^{1}(\mathcal{Q}), g \in L^{2}(\mathcal{Q})$. Since the Biot equations define a PDAE, we combine the original Biot system with the differentiated first equation

$$
-h_{T} \partial_{t} \nabla_{x} \cdot \tilde{\boldsymbol{\sigma}}(\boldsymbol{u}, p)=h_{T} \partial_{t} \boldsymbol{f} .
$$

This last differentiation step is inspired by the differentiation procedure used in DAE (differential algebraic equation) theory in order to obtain underlying ODEs (ordinary differential equations); see, e.g., [35]. To be more precise, we choose a differentiated test function 
$\partial_{t} \boldsymbol{v}_{h}, \boldsymbol{v}_{h} \in \mathcal{V}_{h, r_{u}, r_{T}}$, and multiply the sum of equations (2.3) and (3.3) by this test function. Integration over the whole space-time cylinder yields

$$
\left\langle-\nabla_{x} \cdot(\boldsymbol{\sigma}-b p \boldsymbol{I})-h_{T} \partial_{t}\left[\nabla_{x} \cdot(\boldsymbol{\sigma}-b p \boldsymbol{I})\right], \partial_{t} \boldsymbol{v}_{h}\right\rangle_{\boldsymbol{L}^{2}(\mathcal{Q})}=\left\langle\boldsymbol{f}+h_{T} \partial_{t} \boldsymbol{f}, \partial_{t} \boldsymbol{v}_{h}\right\rangle_{\boldsymbol{L}^{2}(\mathcal{Q})},
$$

and integration by parts along with the Einstein summation convention leads to

$$
\begin{gathered}
\int_{\mathcal{Q}} C_{i j k l} \varepsilon_{k l}\left(\boldsymbol{u}+h_{T} \partial_{t} \boldsymbol{u}\right) \varepsilon_{i j}\left(\partial_{t} \boldsymbol{v}_{h}\right) d \boldsymbol{x} d t-b \int_{\mathcal{Q}}\left(p+h_{T} \partial_{t} p\right) \nabla_{x} \cdot \partial_{t} \boldsymbol{v}_{h} d \boldsymbol{x} d t \\
+\int_{\partial \mathcal{Q}}(\underbrace{(-\boldsymbol{\sigma}+b p \boldsymbol{I})}_{=-\tilde{\boldsymbol{\sigma}}}+h_{T} \partial_{t}(-\boldsymbol{\sigma}+b p \boldsymbol{I})) \cdot \boldsymbol{n}_{x} \partial_{t} \boldsymbol{v}_{h} d s \\
=\left\langle\boldsymbol{f}+h_{T} \partial_{t} \boldsymbol{f}, \partial_{t} \boldsymbol{v}_{h}\right\rangle_{\boldsymbol{L}^{2}(\mathcal{Q})} .
\end{gathered}
$$

On the other hand, the multiplication of the evolution equation (2.4) for the pressure by a time-upwind test function $q_{h}+h_{T} \partial_{t} q_{h}$ gives, again using integration by parts,

$$
\begin{gathered}
\int_{\mathcal{Q}} c_{0} \partial_{t} p\left(q_{h}+h_{T} \partial_{t} q_{h}\right) d \boldsymbol{x} d t+b \int_{\mathcal{Q}} \nabla_{x} \cdot \partial_{t} \boldsymbol{u}\left(q_{h}+h_{T} \partial_{t} q_{h}\right) d \boldsymbol{x} d t \\
-\int_{\partial \mathcal{Q}}\left(\mathcal{K} \nabla_{x} p\left(q_{h}+\partial_{t} q_{h}\right)\right) \cdot \boldsymbol{n}_{x} d s+\int_{\mathcal{Q}} \mathcal{K} \nabla_{x} p \nabla_{x}\left(q_{h}+h_{T} \partial_{t} q_{h}\right) d \boldsymbol{x} d t \\
=\left\langle g, q_{h}+h_{T} \partial_{t} q_{h}\right\rangle_{L^{2}(\mathcal{Q})} .
\end{gathered}
$$

REMARK 3.2. For the derivation above, the product structure of the parametrization and the smoothness of the isogeometric basis functions in each mesh element $\mathcal{K}$ lead to the well-definedness of the mixed derivatives $\partial_{x_{i}} \partial_{t} \boldsymbol{v}_{h}, \partial_{x_{i}} \partial_{t} q_{h}$. The operators $\partial_{x_{i}}, i=1, \ldots, d$, denote the derivatives with respect to the spatial coordinates. Both equations (3.4) and (3.5) are in some sense the blueprints for the next definition.

DEFINITION 3.3 (Discrete variational formulation).

Find $\boldsymbol{u}_{h} \in \mathcal{V}_{h, r_{u}, r_{T}}$ and $p_{h} \in \mathcal{W}_{h, r_{p}, r_{T}}$ such that

$$
\begin{aligned}
\tilde{e}\left(\boldsymbol{u}_{h}+h_{T} \partial_{t} \boldsymbol{u}_{h}, \partial_{t} \boldsymbol{v}_{h}\right) & -b\left\langle p_{h}+h_{T} \partial_{t} p_{h}, \nabla_{x} \cdot \partial_{t} \boldsymbol{v}_{h}\right\rangle_{L^{2}(\mathcal{Q})} \\
=l_{1}\left(\partial_{t} \boldsymbol{v}_{h}\right), & \\
c_{0}\left\langle\partial_{t} p_{h}, q_{h}+h_{T} \partial_{t} q_{h}\right\rangle_{L^{2}(\mathcal{Q})} & +b\left\langle\nabla_{x} \cdot \partial_{t} \boldsymbol{u}_{h}, q_{h}+h_{T} \partial_{t} q_{h}\right\rangle_{L^{2}(\mathcal{Q})} \\
& +\tilde{a}\left(p_{h}, q_{h}+h_{T} \partial_{t} q_{h}\right)=l_{2}\left(q_{h}+h_{T} \partial_{t} q_{h}\right),
\end{aligned}
$$

for all $v_{h} \in \mathcal{V}_{h, r_{u}, r_{T}}$ and $q_{h} \in \mathcal{W}_{h, r_{p}, r_{T}}$,

with the linear forms

$$
\begin{aligned}
l_{1}(\boldsymbol{v}) & :=\left\langle\boldsymbol{f}+h_{T} \partial_{t} \boldsymbol{f}, \boldsymbol{v}\right\rangle_{\boldsymbol{L}^{2}(\mathcal{Q})}+\left\langle\boldsymbol{t}_{n}+h_{T} \partial_{t} \boldsymbol{t}_{n}, \boldsymbol{v}\right\rangle_{\boldsymbol{L}^{2}\left(\Sigma_{t}\right)}, \\
l_{2}(q) & :=\langle g, q\rangle_{L^{2}(\mathcal{Q})}+\left\langle v_{f}, q\right\rangle_{L^{2}\left(\Sigma_{f}\right)},
\end{aligned}
$$

and the bilinear forms

$$
\begin{aligned}
\tilde{e}(\boldsymbol{u}, \boldsymbol{v}) & :=\int_{\mathcal{Q}} C_{i j k l} \varepsilon_{k l}(\boldsymbol{u}) \varepsilon_{i j}(\boldsymbol{v}) d \boldsymbol{x} d t, \\
\tilde{a}(p, q) & :=\left\langle\mathcal{K} \nabla_{x} p, \nabla_{x} q\right\rangle_{\boldsymbol{L}^{2}(\mathcal{Q})} .
\end{aligned}
$$

For later considerations, we use instead of (3.6)-(3.7) the following equivalent formulation: 
Find $\boldsymbol{u}_{h}, p_{h}$ such that

$$
b_{S T}\left(\left[\boldsymbol{u}_{h}, p_{h}\right],\left[\boldsymbol{v}_{h}, q_{h}\right]\right)=l\left(\left[\boldsymbol{v}_{h}, q_{h}\right]\right), \quad \forall \boldsymbol{v}_{h} \in \mathcal{V}_{h, r_{u}, r_{T}}, q_{h} \in \mathcal{W}_{h, r_{p}, r_{T}},
$$

with

$$
\begin{aligned}
& b_{S T}([\boldsymbol{u}, p],[\boldsymbol{v}, q]):=\tilde{e}(\boldsymbol{u}\left.+h_{T} \partial_{t} \boldsymbol{u}, \partial_{t} \boldsymbol{v}\right)-b\left\langle p+h_{T} \partial_{t} p, \nabla_{x} \cdot \partial_{t} \boldsymbol{v}\right\rangle_{L^{2}(\mathcal{Q})} \\
&+c_{0}\left\langle\partial_{t} p, q+h_{T} \partial_{t} q\right\rangle_{L^{2}(\mathcal{Q})} \\
&+b\left\langle\nabla_{x} \cdot \partial_{t} \boldsymbol{u}, q+h_{T} \partial_{t} q\right\rangle_{L^{2}(\mathcal{Q})} \\
&+\tilde{a}\left(p, q+h_{T} \partial_{t} q\right), \\
& l([\boldsymbol{v}, q]):=l_{1}\left(\partial_{t} \boldsymbol{v}\right)+l_{2}\left(q+h_{T} \partial_{t} q\right) .
\end{aligned}
$$

In fact, the variational formulation (3.8) is obtained by adding up the contributions of the two equations (3.6) and (3.7). The discrete formulation is consistent in the following way:

LEMMA 3.4. Assume that there exists a solution to (2.3)-(2.4) satisfying $\boldsymbol{u} \in \boldsymbol{H}^{3}(\mathcal{Q})$ and $p \in H^{2}(\mathcal{Q})$, and let $v_{f} \in L^{2}\left(\Sigma_{f}\right)$ and $\boldsymbol{t}_{n} \in \boldsymbol{L}^{2}\left(\Sigma_{t}\right)$ be the restriction of a function in $\boldsymbol{t}_{e} \in \boldsymbol{H}^{1}(\partial \mathcal{Q})$. Moreover, let $\boldsymbol{f} \in \boldsymbol{H}^{1}(\mathcal{Q}), g \in L^{2}(\mathcal{Q})$. Then

$$
b_{S T}\left([\boldsymbol{u}, p],\left[\boldsymbol{v}_{h}, q_{h}\right]\right)=l\left(\left[\boldsymbol{v}_{h}, q_{h}\right]\right) \quad \text { for all } \boldsymbol{v}_{h} \in \mathcal{V}_{h, r_{u}, r_{T}}, q_{h} \in \mathcal{W}_{h, r_{p}, r_{T}} .
$$

Proof. This is clear due to the equations (3.4) and (3.5), the assumed boundary conditions, and the fact that for the Lipschitz domain $\mathcal{Q}$, the trace operator restricted to $H^{2}(\mathcal{Q})$ defines a linear and continuous operator $\gamma_{0}: H^{2}(\mathcal{Q}) \rightarrow H^{1}(\partial \mathcal{Q})$; see, e.g., [19].

3.3. Uniqueness and existence of the IGA solution. Next, we investigate whether there exists a unique solution to the discrete problem (3.8). For this purpose we prove the coercivity of the bilinear form $b_{S T}(\cdot, \cdot)$ with respect to the space $\mathcal{V}_{h, r_{u}, r_{T}} \times \mathcal{W}_{h, r_{p}, r_{T}}$ endowed with the auxiliary norm

$$
\begin{aligned}
& \|[\boldsymbol{v}, q]\|_{h}^{2}:=h_{T}\left\|\partial_{t} \boldsymbol{v}\right\|_{\mathcal{H}^{1}(\mathcal{Q})}^{2}+\|\boldsymbol{v}\|_{\boldsymbol{H}^{1}\left(\Sigma_{T}\right)}^{2}+h_{T} c_{0}\left\|\partial_{t} q\right\|_{L^{2}(\mathcal{Q})}^{2} \\
& +c_{0}\|q\|_{L^{2}\left(\Sigma_{T}\right)}^{2}+\left\|\nabla_{x} q\right\|_{L^{2}(\mathcal{Q})}^{2}, \quad \text { where } \\
& \|\boldsymbol{v}\|_{\mathcal{H}^{1}(\mathcal{Q})}^{2}:=\int_{\mathcal{Q}} \sum_{i, j}\left(\partial_{x_{i}} v_{j}\right)^{2} d \boldsymbol{x} d t+\|\boldsymbol{v}\|_{\boldsymbol{L}^{2}(\mathcal{Q})}^{2}, \quad \boldsymbol{v}=\left(v_{1}, \ldots, v_{d}\right),
\end{aligned}
$$

and where $\Sigma_{T}=\Omega \times\{T\}$. Exploiting continuity, the piecewise smoothness, and the boundary conditions of the test functions, one can verify easily that $\|[\cdot, \cdot]\|_{h}$ is indeed a norm in $\mathcal{V}_{h, r_{u}, r_{T}} \times \mathcal{W}_{h, r_{p}, r_{T}}$. Before we prove coercivity, we insert here two auxiliary results.

LEMMA 3.5. Let the elasticity tensor $C$ satisfy

$$
\mu(\boldsymbol{C}) \sum_{i, j} x_{i j}^{2} \leq \sum_{i, j, k, l} C_{i j k l} x_{i j} x_{k l}
$$

for all $x_{m n} \in \mathbb{R}, m, n \in\{1, \ldots, d\}$, and some constant $0<\mu(\boldsymbol{C})$. Then the bilinear form

$$
e(\cdot, \cdot): \boldsymbol{V} \times \boldsymbol{V} \rightarrow \mathbb{R},(\boldsymbol{u}, \boldsymbol{v}) \mapsto \int_{\Omega} C_{i j k l} \varepsilon_{k l}(\boldsymbol{u}) \varepsilon_{i j}(\boldsymbol{v}) d \boldsymbol{x}
$$

is symmetric and coercive with respect to the $\boldsymbol{H}^{1}$-norm in $\boldsymbol{V}:=\left\{\boldsymbol{v} \in \boldsymbol{H}^{1}(\Omega) \mid \boldsymbol{v}=0\right.$ on $\left.\Gamma_{u}\right\}$. Thus, there exists a constant $0<c_{e}$ such that

$$
e(\boldsymbol{v}, \boldsymbol{v}) \geq c_{e}\|\boldsymbol{v}\|_{\boldsymbol{H}^{1}(\Omega)}^{2}, \quad \forall \boldsymbol{v} \in \boldsymbol{V} .
$$

The constant depends only on $\Omega, \Gamma_{u}$, and $\boldsymbol{C}$. 
Proof. The inequality (3.11) follows from [1, Corollary 5.9] for both cases $d=2,3$. The one-dimensional case is a consequence of the Poincaré inequality; see [20, Example 3]. The symmetry of $e(\cdot, \cdot)$ is obvious due to the symmetry properties of the elasticity tensor.

Lemma 3.6. There exist constants $0<c_{a}, C_{a}<\infty$ that depend only on $\mathcal{K}, \Gamma_{p}$, and $\Omega$ such that

$$
a(q, q) \geq c_{a}\|q\|_{H^{1}(\Omega)}^{2} \quad \text { and } \quad|a(p, q)| \leq C_{a}\|p\|_{H^{1}(\Omega)}\|q\|_{H^{1}(\Omega)},
$$

for all $p, q \in W:=\left\{w \in H^{1}(\Omega) \mid w=0\right.$ on $\left.\Gamma_{p}\right\}$ and with

$$
a: W \times W \rightarrow \mathbb{R}, \quad(p, q) \mapsto\left\langle\mathcal{K} \nabla_{x} p, \nabla_{x} q\right\rangle_{\mathbf{L}^{2}(\Omega)} .
$$

Proof. An application of the Poincaré inequality and the assumption that the $\mathcal{K}(\boldsymbol{x})$ are uniformly elliptic and bounded symmetric positive definite matrices yields the assertion.

REMARK 3.7. In the context of the space-time discretization, we interpret the derivatives $\partial_{x_{i}}, \partial_{t}$ etc., as weak derivatives with respect to the domain $\mathcal{Q}$. Nevertheless, due to the piecewise smoothness of the test functions and their continuity, we obtain that the weak derivatives are in fact piecewisely defined classical derivatives, and in particular one can assume for $s \in[0, T]$ that $\boldsymbol{v}_{h}(\cdot, s) \in \boldsymbol{V}$ and $q_{h}(\cdot, s) \in W$. The product structure of the parametrization and hence of the test spaces further gives us that $\partial_{t} \boldsymbol{v}_{h}(\cdot, s) \in \boldsymbol{V}$ and $\partial_{t} q_{h}(\cdot, s) \in W$; see (3.2).

Now we arrive at the mentioned coercivity result.

LEMMA 3.8. The bilinear form $b_{S T}$ defined by (3.9) is coercive in the sense that there exists a constant $0<\mu_{c}$ independent of the mesh sizes such that

$$
\mu_{c}\left\|\left[\boldsymbol{u}_{h}, p_{h}\right]\right\|_{h}^{2} \leq b_{S T}\left(\left[\boldsymbol{u}_{h}, p_{h}\right],\left[\boldsymbol{u}_{h}, p_{h}\right]\right), \quad \forall\left[\boldsymbol{u}_{h}, p_{h}\right] \in \mathcal{V}_{h, r_{u}, r_{T}} \times \mathcal{W}_{h, r_{p}, r_{T}} .
$$

The constant $\mu_{c}$ can be chosen independently of $c_{0}$.

Proof. For reasons of clarity we estimate the different terms in the definition of $b_{S T}$, i.e., (3.9), separately, starting with the non-mixed terms in which either only $\boldsymbol{u}$ and $\boldsymbol{v}$ or only $p, q$ occur.

1. Consider the term $\tilde{e}(\cdot, \cdot)$, and note that $\boldsymbol{u}_{h}(\cdot, t=0)=\mathbf{0}$. Observe the symmetry of the bilinear form $\tilde{e}(\cdot, \cdot)$, which is obvious by the symmetry of $e(\cdot, \cdot)$; see Lemma 3.5. By means of these properties and Green's formula we can write

$$
\begin{aligned}
\tilde{e}\left(\boldsymbol{u}_{h}, \partial_{t} \boldsymbol{u}_{h}\right) & =\int_{\mathcal{Q}} C_{i j k l} \varepsilon_{k l}\left(\boldsymbol{u}_{h}\right) \varepsilon_{i j}\left(\partial_{t} \boldsymbol{u}_{h}\right) d \boldsymbol{x} d t= \\
& =\frac{1}{2} \int_{\mathcal{Q}} \partial_{t}\left(C_{i j k l} \varepsilon_{k l}\left(\boldsymbol{u}_{h}\right) \varepsilon_{i j}\left(\boldsymbol{u}_{h}\right)\right) d t d \boldsymbol{x} \\
& =\frac{1}{2} \int_{\partial \mathcal{Q}} C_{i j k l} \varepsilon_{k l}\left(\boldsymbol{u}_{h}\right) \varepsilon_{i j}\left(\boldsymbol{u}_{h}\right) \cdot n_{t} d s \\
& =\frac{1}{2} \int_{L^{2}\left(\Sigma_{T}\right)} C_{i j k l} \varepsilon_{k l}\left(\boldsymbol{u}_{h}\right) \varepsilon_{i j}\left(\boldsymbol{u}_{h}\right) d \boldsymbol{x} \\
& =\frac{1}{2} e\left(\boldsymbol{u}_{h}(\cdot, T), \boldsymbol{u}_{h}(\cdot, T)\right) \geq \frac{c_{e}}{2}\left\|\boldsymbol{u}_{h}(\cdot, T)\right\|_{\boldsymbol{H}^{1}(\Omega)}^{2} .
\end{aligned}
$$

Here we used the piecewise smoothness of the test functions, i.e., $\boldsymbol{u}_{h}(\cdot, t) \in \boldsymbol{V}$, and the coercivity of the elasticity form $e(\cdot, \cdot)$ (Lemma 3.5$)$ with $\left(\boldsymbol{n}_{x}, n_{t}\right)$ denoting the outer unit normal vector of the space-time domain. 
2. In view of Remark 3.7 and the coercivity of $e(\cdot, \cdot)$, it holds that

$$
\begin{aligned}
h_{T} \tilde{e}\left(\partial_{t} \boldsymbol{u}_{h}, \partial_{t} \boldsymbol{u}_{h}\right) & =h_{T} \int_{\mathcal{Q}} C_{i j k l} \varepsilon_{k l}\left(\partial_{t} \boldsymbol{u}_{h}\right) \varepsilon_{i j}\left(\partial_{t} \boldsymbol{u}_{h}\right) d \boldsymbol{x} d t \\
& =h_{T} \int_{0}^{T} e\left(\partial_{t} \boldsymbol{u}_{h}(\cdot, t), \partial_{t} \boldsymbol{u}_{h}(\cdot, t)\right) d t \\
& \geq h_{T} \int_{0}^{T} c_{e}\left\|\partial_{t} \boldsymbol{u}_{h}(\cdot, t)\right\|_{\boldsymbol{H}^{1}(\Omega)}^{2} d t \\
& =h_{T} c_{e} \int_{0}^{T} \int_{\Omega} \sum_{i, j}\left(\partial_{x_{i}} \partial_{t} u_{h, j}\right)^{2}+\sum_{j}\left(\partial_{t} u_{h, j}\right)^{2} d \boldsymbol{x} d t \\
& =h_{T} c_{e}\left\|\partial_{t} \boldsymbol{u}_{h}\right\|_{\mathcal{H}^{1}(\mathcal{Q})}^{2} .
\end{aligned}
$$

One notices Fubini's theorem and the notation $u_{h, i}$ for the $i$-th component of $\boldsymbol{u}_{h}$.

3. By the chain rule and the zero initial conditions, we get

$$
\left\langle c_{0} \partial_{t} p_{h}, p_{h}\right\rangle_{L^{2}(\mathcal{Q})}=\int_{\mathcal{Q}} \frac{c_{0}}{2} \partial_{t}\left(p_{h}^{2}\right) d t d \boldsymbol{x}=\frac{c_{0}}{2} \int_{\partial \mathcal{Q}} p_{h}^{2} \cdot n_{t} d s=\frac{c_{0}}{2}\left\|p_{h}\right\|_{L^{2}\left(\Sigma_{T}\right)}^{2} .
$$

4. Obviously,

$$
h_{T}\left\langle c_{0} \partial_{t} p_{h}, \partial_{t} p_{h}\right\rangle_{L^{2}(\mathcal{Q})}=h_{T} c_{0}\left\|\partial_{t} p_{h}\right\|_{L^{2}(\mathcal{Q})}^{2} .
$$

5. Moreover, with Remark 3.7 as well as with Lemma 3.6 and Fubini's theorem, one can estimate

$$
\begin{aligned}
\tilde{a}\left(p_{h}, p_{h}\right)=\int_{\Omega} \int_{0}^{T} \mathcal{K} \nabla_{x} p_{h} \nabla_{x} p_{h} d t d \boldsymbol{x} & =\int_{0}^{T} a\left(p_{h}(\cdot, t), p_{h}(\cdot, t)\right) d t \\
& \geq c_{a}\left\|\nabla_{x} p_{h}\right\|_{\boldsymbol{L}^{2}(\mathcal{Q})}^{2} .
\end{aligned}
$$

6. Finally, by the symmetry of $a(\cdot, \cdot)$, the last non-mixed term yields

$$
\begin{aligned}
h_{T} \tilde{a}\left(p_{h}, \partial_{t} p_{h}\right) & =h_{T} \int_{0}^{T} a\left(p_{h}(\cdot, t), \partial_{t} p_{h}(\cdot, t)\right) d t \\
& =\frac{h_{T}}{2} \int_{0}^{T} \partial_{t} a\left(p_{h}(\cdot, t), p_{h}(\cdot, t)\right) d t=\frac{h_{T}}{2} a\left(p_{h}(\cdot, T), p_{h}(\cdot, T)\right) \geq 0 .
\end{aligned}
$$

For the first, third, and last point above, we used the assumption that $p_{h}=0, \boldsymbol{u}_{h}=0$ on $\Sigma_{0}$. Next we sum up all the remaining terms in the definition of $b_{S T}\left(\left[\boldsymbol{u}_{h}, p_{h}\right],\left[\boldsymbol{u}_{h}, p_{h}\right]\right)$, i.e., in the sum of the right-hand side of (3.9). We get

$$
\begin{aligned}
b\left[-\left\langle p_{h}, \nabla_{x} \cdot\right.\right. & \left.\partial_{t} \boldsymbol{u}_{h}\right\rangle_{L^{2}(\mathcal{Q})}-h_{T}\left\langle\partial_{t} p_{h}, \nabla_{x} \cdot \partial_{t} \boldsymbol{u}_{h}\right\rangle_{L^{2}(\mathcal{Q})} \\
& \left.+\left\langle\nabla_{x} \cdot \partial_{t} \boldsymbol{u}_{h}, p_{h}\right\rangle_{L^{2}(\mathcal{Q})}+h_{T}\left\langle\nabla_{x} \cdot \partial_{t} \boldsymbol{u}_{h}, \partial_{t} p_{h}\right\rangle_{L^{2}(\mathcal{Q})}\right]=0 .
\end{aligned}
$$

Thus, the mixed terms vanish. So it is obvious by the above estimates that

$$
\begin{aligned}
b_{S T}\left(\left[\boldsymbol{u}_{h}, p_{h}\right],\left[\boldsymbol{u}_{h}, p_{h}\right]\right) \geq & \mu_{c}\left(\left\|\boldsymbol{u}_{h}\right\|_{\boldsymbol{H}^{1}\left(\Sigma_{T}\right)}^{2}\right. \\
& +h_{T}\left\|\partial_{t} \boldsymbol{u}_{h}\right\|_{\mathcal{H}^{1}(\mathcal{Q})}^{2}+c_{0}\left\|p_{h}\right\|_{L^{2}\left(\Sigma_{T}\right)}^{2} \\
& \left.+h_{T} c_{0}\left\|\partial_{t} p_{h}\right\|_{L^{2}(\mathcal{Q})}^{2}+\left\|\nabla_{x} p_{h}\right\|_{\boldsymbol{L}^{2}(\mathcal{Q})}^{2}\right) \\
& =\mu_{c}\left\|\left[\boldsymbol{u}_{h}, p_{h}\right]\right\|_{h}^{2},
\end{aligned}
$$

for $\mu_{c}:=\min \left\{\frac{c_{e}}{2}, c_{a}, \frac{1}{2}\right\}$. 
If we now choose bases $\left\{\psi_{i} \mid i=1, \ldots, N_{h}^{p}\right\}$ and $\left\{\phi_{j} \mid j=1, \ldots, N_{h}^{u}\right\}$ of the test spaces $\mathcal{W}_{h, r_{p}, r_{T}}$ and $\mathcal{V}_{h, r_{u}, r_{T}}$, respectively, then the coefficient vectors $P^{h}:=\left(\hat{p}_{1}, \ldots, \hat{p}_{N_{h}^{p}}\right)$, $U^{h}:=\left(\hat{u}_{1}, \ldots, \hat{u}_{N_{h}^{u}}\right)$, with

$$
\boldsymbol{u} \approx \boldsymbol{u}_{h}:=\sum_{j=1}^{N_{h}^{u}} \hat{u}_{j} \phi_{j}, \quad p \approx p_{h}:=\sum_{i=1}^{N_{h}^{p}} \hat{p}_{i} \psi_{i},
$$

which define the discretized solution $\boldsymbol{u}_{h}, p_{h}$, are obtained by solving one linear system of the type

$$
S^{h}\left[\begin{array}{l}
U^{h} \\
P^{h}
\end{array}\right]=\left[\begin{array}{l}
R^{1} \\
R^{2}
\end{array}\right]
$$

The shown coercivity of the bilinear form $b_{S T}$ implies the positive definiteness of the system matrix $S^{h}$. Thus, the existence of a unique solution is clear.

THEOREM 3.9 (Uniqueness and existence of the IGA solution). There exists a unique solution to the variational problem (3.8).

The latter theorem guarantees the well-definedness of our numerical scheme, but for a useful method, a convergence statement is an important aspect too. Consequently, we face this issue in the next part.

4. Discretization error analysis. The main objective of this section is the derivation of an a priori discretization error estimate for the numerical approximation of the displacement $u$ and the pressure $p$ in the setting of Lemma 3.4. For reasons of simplification, we set in the whole section without loss of generalty $b=1$ and remark that $0.5 \leq b \leq 1$ in most applications. We start with a result from the IGA theory that will be used below.

LEMMA 4.1 (Inverse inequality). Let the space-time mesh be regular with polynomial degrees $r_{T}, r_{S}$ greater than zero. Then, for $i=1, \ldots, d$, it holds that

$$
\begin{aligned}
h_{T}\left\|\partial_{t} \partial_{x_{i}} v_{h}\right\|_{L^{2}(\mathcal{Q})} & \leq C_{i n v, 1}\left\|\partial_{x_{i}} v_{h}\right\|_{L^{2}(\mathcal{Q})}, \\
h_{T}\left\|\partial_{t} v_{h}\right\|_{L^{2}(\mathcal{Q})} & \leq C_{i n v, 2}\left\|v_{h}\right\|_{L^{2}(\mathcal{Q})}, \quad v_{h} \in \mathcal{V}_{h, r_{S}, r_{T}} \cap C^{0}(\mathcal{Q}),
\end{aligned}
$$

where $C_{i n v, j}$ are constants independent of the mesh sizes and $v_{h}$.

Proof. We remark that $\partial_{x_{i}} v_{h}$ is piecewise smooth and that for $\boldsymbol{x} \in \mathcal{Q}$ the function $\partial_{x_{i}} v_{h}(\boldsymbol{x}, \cdot)$ is continuous in time. By the product structure of the space $\mathcal{V}_{h, \tilde{\boldsymbol{r}}}$ and $\mathcal{Q}$, one sees that $\partial_{x_{i}} v_{h}(\boldsymbol{x}, \cdot)$ is an element of a univariate spline space $V_{h_{T}, r_{T}}$ with mesh size $h_{T}$. Due to the regularity of the mesh and as a consequence of [5, Theorem 4.2], we find a constant $C$ independent of $\boldsymbol{x}$ such that the estimate

$$
h_{T}\left\|\partial_{t} \partial_{x_{i}} v_{h}(\boldsymbol{x}, \cdot)\right\|_{L^{2}((0, T))} \leq C\left\|\partial_{x_{i}} v_{h}(\boldsymbol{x}, \cdot)\right\|_{L^{2}((0, T))} .
$$

is fulfilled. Integration over $\Omega$ yields the assertion for inequality (4.1). The second estimate (4.2) can be proven analogously.

Next we define the auxiliary spaces

$$
\begin{aligned}
\mathcal{V}_{0} & :=\left\{\boldsymbol{v}=\left(v_{1}, \ldots, v_{d}\right) \in \boldsymbol{H}^{1}(\mathcal{Q}) \mid \nabla_{x} \partial_{t} v_{i} \in \boldsymbol{L}^{2}(\mathcal{Q}), v_{i}=0 \text { on } \Sigma_{u} \cup \Sigma_{0}, \forall i\right\}, \\
\mathcal{W}_{0} & :=\left\{q \in H^{1}(\mathcal{Q}) \mid \nabla_{x} \partial_{t} q \in \boldsymbol{L}^{2}(\mathcal{Q}), q=0 \text { on } \Sigma_{p} \cup \Sigma_{0}\right\}
\end{aligned}
$$

and a corresponding norm

$$
\begin{aligned}
\|[\boldsymbol{u}, p]\|_{h, \star}^{2}:=\left\|\partial_{t} \boldsymbol{u}\right\|_{\mathcal{H}^{1}(\mathcal{Q})}^{2} & +\frac{1}{h_{T}}\left(\|\boldsymbol{u}\|_{\mathcal{H}^{1}(\mathcal{Q})}^{2}+\|p\|_{L^{2}(\mathcal{Q})}^{2}\right) \\
& +\left\|\partial_{t} p\right\|_{L^{2}(\mathcal{Q})}^{2}+\left\|\nabla_{x} p\right\|_{\boldsymbol{L}^{2}(\mathcal{Q})}^{2}
\end{aligned}
$$


and we note the well-definedness of the terms on the right-hand side of (3.9) provided that $\boldsymbol{u}$, $\boldsymbol{v} \in \mathcal{V}_{0}$, and $p, q \in \mathcal{W}_{0}$. Using the latter norm we can state now a boundedness result for $b_{S T}$.

LEMMA 4.2. The bilinear form $b_{S T}$ is continuous with respect to the norms $\|\cdot\|_{h}$ and $\|\cdot\|_{h, \star}$ in the sense that

$$
b_{S T}\left([\boldsymbol{u}, p],\left[\boldsymbol{u}_{h}, p_{h}\right]\right) \leq \mu_{b}\|[\boldsymbol{u}, p]\|_{h, \star}\left\|\left[\boldsymbol{u}_{h}, p_{h}\right]\right\|_{h}
$$

for all $[\boldsymbol{u}, p] \in \mathcal{V}_{0} \times \mathcal{W}_{0}$ and $\left[\boldsymbol{u}_{h}, p_{h}\right] \in \mathcal{V}_{h, r_{u}, r_{T}} \times \mathcal{W}_{h, r_{p}, r_{T}}$ and some constant $\mu_{b}$ independent of $c_{0}$ and the mesh size.

Proof. We first look at the different terms appearing in the definition of $b_{S T}$ and estimate them separately. Doing so, we also introduce some auxiliary constants $C_{1}, \ldots, C_{8}$.

1. By the definition of the elasticity bilinear form $\tilde{e}(\cdot, \cdot)$ (see Definition 3.3) and the Cauchy-Schwarz inequality, we have

$$
\begin{aligned}
S_{1}\left(\boldsymbol{u}, p, \boldsymbol{u}_{h}, p_{h}\right) & :=\tilde{e}\left(\boldsymbol{u}, \partial_{t} \boldsymbol{u}_{h}\right)=\int_{\mathcal{Q}} C_{i j k l} \boldsymbol{\epsilon}_{k l}(\boldsymbol{u}) \boldsymbol{\epsilon}_{i j}\left(\partial_{t} \boldsymbol{u}_{h}\right) d \boldsymbol{x} d t \\
& =\int_{\mathcal{Q}} \frac{1}{4} C_{i j k l}\left(\partial_{x_{k}} u_{l} \partial_{x_{i}} \partial_{t} u_{h, j}+\partial_{x_{l}} u_{k} \partial_{x_{i}} \partial_{t} u_{h, j}\right. \\
& \left.\quad+\partial_{x_{k}} u_{l} \partial_{x_{j}} \partial_{t} u_{h, i}+\partial_{x_{l}} u_{k} \partial_{x_{j}} \partial_{t} u_{h, i}\right) d \boldsymbol{x} d t \\
& \leq C_{\tilde{e}} \frac{1}{\sqrt{h_{T}}}\|\boldsymbol{u}\|_{\mathcal{H}^{1}(\mathcal{Q})} \sqrt{h_{T}}\left\|\partial_{t} \boldsymbol{u}_{h}\right\|_{\mathcal{H}^{1}(\mathcal{Q})} \\
& \leq C_{1}\|[\boldsymbol{u}, p]\|_{h, \star}\left\|\left[\boldsymbol{u}_{h}, p_{h}\right]\right\|_{h} .
\end{aligned}
$$

Above we can set $C_{\tilde{e}}:=\sum_{i, j, k, l}\left|C_{i j k l}\right|$. Here, $u_{i}$ denotes the $i$-th component of $\boldsymbol{u}$. 2. The Cauchy-Schwarz inequality yields

$$
\begin{aligned}
S_{2}\left(\boldsymbol{u}, p, \boldsymbol{u}_{h}, p_{h}\right) & :=-\left\langle p, \nabla_{x} \cdot \partial_{t} \boldsymbol{u}_{h}\right\rangle_{L^{2}(\mathcal{Q})} \leq\|p\|_{L^{2}(\mathcal{Q})}\left\|\nabla_{x} \cdot \partial_{t} \boldsymbol{u}_{h}\right\|_{L^{2}(\mathcal{Q})} \\
& \leq \frac{1}{\sqrt{h_{T}}}\|p\|_{L^{2}(\mathcal{Q})} \sqrt{3 h_{T}}\left\|\partial_{t} \boldsymbol{u}_{h}\right\|_{\mathcal{H}^{1}(\mathcal{Q})} \\
& \leq C_{2}\|[\boldsymbol{u}, p]\|_{h, \star}\left\|\left[\boldsymbol{u}_{h}, p_{h}\right]\right\|_{h} .
\end{aligned}
$$

3. In an analogous manner to the first point it holds that

$$
\begin{aligned}
S_{3}\left(\boldsymbol{u}, p, \boldsymbol{u}_{h}, p_{h}\right):=h_{T} \tilde{e}\left(\partial_{t} \boldsymbol{u}, \partial_{t} \boldsymbol{u}_{h}\right) & \leq C_{\tilde{e}} h_{T}\left\|\partial_{t} \boldsymbol{u}\right\|_{\mathcal{H}^{1}(\mathcal{Q})}\left\|\partial_{t} \boldsymbol{u}_{h}\right\|_{\mathcal{H}^{1}(\mathcal{Q})} \\
& \leq C_{\tilde{e}} \sqrt{h_{T}}\left\|\partial_{t} \boldsymbol{u}\right\|_{\mathcal{H}^{1}(\mathcal{Q})} \sqrt{h_{T}}\left\|\partial_{t} \boldsymbol{u}_{h}\right\|_{\mathcal{H}^{1}(\mathcal{Q})} \\
& \leq C_{3}\|[\boldsymbol{u}, p]\|_{h, \star}\left\|\left[\boldsymbol{u}_{h}, p_{h}\right]\right\|_{h} .
\end{aligned}
$$

4. A further term can be bounded similarly as in the second point:

$$
\begin{aligned}
S_{4}\left(\boldsymbol{u}, p, \boldsymbol{u}_{h}, p_{h}\right) & :=-h_{T}\left\langle\partial_{t} p, \nabla_{x} \cdot \partial_{t} \boldsymbol{u}_{h}\right\rangle_{L^{2}(\mathcal{Q})} \\
& \leq h_{T}\left\|\partial_{t} p\right\|_{L^{2}(\mathcal{Q})}\left\|\nabla_{x} \cdot \partial_{t} \boldsymbol{u}_{h}\right\|_{L^{2}(\mathcal{Q})} \\
& \leq \sqrt{h_{T}}\left\|\partial_{t} p\right\|_{L^{2}(\mathcal{Q})} \sqrt{3 h_{T}}\left\|\partial_{t} \boldsymbol{u}_{h}\right\|_{\mathcal{H}^{1}(\mathcal{Q})} \\
& \leq C_{4}\|[\boldsymbol{u}, p]\|_{h, \star}\left\|\left[\boldsymbol{u}_{h}, p_{h}\right]\right\|_{h}
\end{aligned}
$$


5. Then we have by the assumption $c_{0} \leq 1$ and by means of the Poincaré inequality (see, e.g., [20, Example 3]) for some constant $C_{P}$ that

$$
\begin{aligned}
S_{5}\left(\boldsymbol{u}, p, \boldsymbol{u}_{h}, p_{h}\right) & :=\left\langle c_{0} \partial_{t} p+\nabla_{x} \cdot \partial_{t} \boldsymbol{u}, p_{h}\right\rangle_{L^{2}(\mathcal{Q})} \\
& \leq\left(c_{0}\left\|\partial_{t} p\right\|_{L^{2}(\mathcal{Q})}+\sqrt{3}\left\|\partial_{t} \boldsymbol{u}\right\|_{\mathcal{H}^{1}(\mathcal{Q})}\right)\left\|p_{h}\right\|_{L^{2}(\mathcal{Q})} \\
& \leq C_{P}\left(c_{0}\left\|\partial_{t} p\right\|_{L^{2}(\mathcal{Q})}+\sqrt{3}\left\|\partial_{t} \boldsymbol{u}\right\|_{\mathcal{H}^{1}(\mathcal{Q})}\right)\left\|\nabla_{x} p_{h}\right\|_{\boldsymbol{L}^{2}(\mathcal{Q})} \\
& \leq C_{5}\|[\boldsymbol{u}, p]\|_{h, \star}\left\|\left[\boldsymbol{u}_{h}, p_{h}\right]\right\|_{h} .
\end{aligned}
$$

6. Since $\mathcal{K}$ is a symmetric positive-definite matrix, one further obtains by Definition 3.3 that

$$
\begin{aligned}
S_{6}\left(\boldsymbol{u}, p, \boldsymbol{u}_{h}, p_{h}\right):=\tilde{a}\left(p, p_{h}\right) & \leq \mu_{K}\left\|\nabla_{x} p\right\|_{\boldsymbol{L}^{2}(\mathcal{Q})}\left\|\nabla_{x} p_{h}\right\|_{\boldsymbol{L}^{2}(\mathcal{Q})} \\
& \leq C_{6}\|[\boldsymbol{u}, p]\|_{h, \star}\left\|\left[\boldsymbol{u}_{h}, p_{h}\right]\right\|_{h},
\end{aligned}
$$

where the positive number $\mu_{K}<\infty$ is the supremum over all eigenvalues of the matrices $\mathcal{K}(\boldsymbol{x})$.

7. We proceed with the seventh term. Again the Cauchy-Schwarz inequality along with (4.2) yields

$$
\begin{aligned}
S_{7}\left(\boldsymbol{u}, p, \boldsymbol{u}_{h}, p_{h}\right): & =h_{T}\left\langle c_{0} \partial_{t} p+\nabla_{x} \cdot \partial_{t} \boldsymbol{u}, \partial_{t} p_{h}\right\rangle_{L^{2}(\mathcal{Q})} \\
\leq & c_{0} \sqrt{h_{T}}\left\|\partial_{t} p\right\|_{L^{2}(\mathcal{Q})} \sqrt{h_{T}}\left\|\partial_{t} p_{h}\right\|_{L^{2}(\mathcal{Q})} \\
& +\left\|\nabla_{x} \cdot \partial_{t} \boldsymbol{u}\right\|_{L^{2}(\mathcal{Q})} h_{T}\left\|\partial_{t} p_{h}\right\|_{L^{2}(\mathcal{Q})} \\
\leq & \sqrt{c_{0} h_{T}}\left\|\partial_{t} p\right\|_{L^{2}(\mathcal{Q})} \sqrt{c_{0} h_{T}}\left\|\partial_{t} p_{h}\right\|_{L^{2}(\mathcal{Q})} \\
& \quad+\sqrt{3}\left\|\partial_{t} \boldsymbol{u}\right\|_{\mathcal{H}^{1}(\mathcal{Q})} C_{i n v, 2}\left\|p_{h}\right\|_{L^{2}(\mathcal{Q})} \\
\leq & C_{7}\|[\boldsymbol{u}, p]\|_{h, \star}\left\|\left[\boldsymbol{u}_{h}, p_{h}\right]\right\|_{h} .
\end{aligned}
$$

Note that in order to obtain the last inequality sign, we again applied the Poincaré inequality $\left\|p_{h}\right\|_{L^{2}(\mathcal{Q})} \leq C_{P}\left\|\nabla_{x} p_{h}\right\|_{L^{2}(\mathcal{Q})}$.

8. Finally, for the last term and with $\mu_{K}$ defined in point 6 , we have

$$
\begin{aligned}
S_{8}\left(\boldsymbol{u}, p, \boldsymbol{u}_{h}, p_{h}\right) & :=h_{T} \tilde{a}\left(p, \partial_{t} p_{h}\right) \\
& \leq h_{T} \mu_{K}\left\|\nabla_{x} p\right\|_{\boldsymbol{L}^{2}(\mathcal{Q})}\left\|\nabla_{x} \partial_{t} p_{h}\right\|_{\boldsymbol{L}^{2}(\mathcal{Q})} \\
& \leq \mu_{K} C_{i n v, 1}\left\|\nabla_{x} p\right\|_{\boldsymbol{L}^{2}(\mathcal{Q})}\left\|\nabla_{x} p_{h}\right\|_{\boldsymbol{L}^{2}(\mathcal{Q})} \\
& \leq C_{8}\|[\boldsymbol{u}, p]\|_{h, \star}\left\|\left[\boldsymbol{u}_{h}, p_{h}\right]\right\|_{h} .
\end{aligned}
$$

Here the forelast inequality sign follows by using the adapted inverse estimate (4.2) in Lemma 4.1 and the regular mesh assumption.

Summarizing, we obtain the original form $b_{S T}$ as the sum of the different terms $S_{i}$, i.e.,

$$
\sum_{i=1}^{8} S_{i}\left(\boldsymbol{u}, p, \boldsymbol{u}_{h}, p_{h}\right)=b_{S T}\left([\boldsymbol{u}, p],\left[\boldsymbol{u}_{h}, p_{h}\right]\right) .
$$

By adding up the above inequalities, the statement follows with $\mu_{b}:=\sum_{i} C_{i}$.

Next we introduce NURBS projections, i.e., projections onto NURBS spaces, which can be used to measure the approximation properties of the test function spaces.

LemmA 4.3. Let $v \in H^{s}(\mathcal{Q}), s \geq \max \left\{s_{1}, s_{2}\right\}$, with $1 \leq s_{1} \leq r_{T}+1,1 \leq s_{2} \leq r_{S}+1$, and $\mathcal{V}_{h, r_{S}, r_{T}}$ the space-time NURBS space with an underlying regular mesh. 


\section{ETNA}

Kent State University and

Johann Radon Institute (RICAM)

Then there exists a projection $\Pi_{h}: H^{1}(\mathcal{Q}) \rightarrow \mathcal{V}_{h, r_{S}, r_{T}}$ and constants $C_{\Pi, j}$ not depending on $h_{S}, h_{T}$, and $v$ such that

$$
\begin{aligned}
\left\|\Pi_{h} v-v\right\|_{L^{2}(\mathcal{Q})} & \leq C_{\Pi, 1}\left(h_{T}^{s_{1}}+h_{S}^{s_{2}}\right)\|v\|_{H^{s}(\mathcal{Q})} \\
\left\|\partial_{x_{i}}\left(\Pi_{h} v-v\right)\right\|_{L^{2}(\mathcal{Q})} & \leq C_{\Pi, 2}\left(h_{T}^{s_{1}-1}+h_{S}^{s_{2}-1}\right)\|v\|_{H^{s}(\mathcal{Q})} \\
\left\|\partial_{t}\left(\Pi_{h} v-v\right)\right\|_{L^{2}(\mathcal{Q})} & \leq C_{\Pi, 3}\left(h_{T}^{s_{1}-1}+h_{S}^{s_{2}-1}\right)\|v\|_{H^{s}(\mathcal{Q})}
\end{aligned}
$$

If additionally $s \geq \max \left\{s_{1}+1, s_{2}+1\right\}$, it moreover holds that

$$
\begin{aligned}
\left\|\partial_{x_{i}} \partial_{t}\left(\Pi_{h} v-v\right)\right\|_{L^{2}(\mathcal{Q})} & \leq C_{\Pi, 4}\left(h_{T}^{s_{1}-1}+h_{S}^{s_{2}-1}\right)\|v\|_{H^{s}(\mathcal{Q})}, \\
\left\|\partial_{x_{i}}\left(\Pi_{h} v-v\right)\right\|_{L^{2}(\mathcal{Q})} & \leq C_{\Pi, 5}\left(h_{T}^{s_{1}}+h_{S}^{s_{2}-1}\right)\|v\|_{H^{s}(\mathcal{Q})} .
\end{aligned}
$$

The approximation results are also valid in case of homogeneous Dirichlet boundary conditions on the whole or on a part of the boundary.

Proof. In the following, $C$ denotes a constant that depends only on the parametrization but may change at different occurrences. The main idea of the proof is the application of IGA approximation results presented in [14, Part 3]. For a better understanding we define the auxiliary derivatives

$$
D_{\tilde{\boldsymbol{\Phi}}}^{s} u:=\left(\partial_{\zeta_{1}}^{s_{1}} \ldots \partial_{\zeta_{d}}^{s_{d}} \partial_{\zeta_{d+1}}^{s_{d+1}}(u \circ \tilde{\boldsymbol{\Phi}})\right) \circ \tilde{\boldsymbol{\Phi}}^{-1}, \quad \boldsymbol{s}=\left(s_{1}, \ldots, s_{d+1}\right) \in \mathbb{N}^{d+1},
$$

for sufficiently regular mappings $u$. Here $\partial_{\zeta_{i}}$ stands for the derivative with respect to the $i$-th coordinate in the parametric domain $\hat{\mathcal{Q}}$. The latter definition is analogous to $[14,(56)$ in Part 3]. Let $v \in H^{2}(\mathcal{K})$ and $\mathcal{K}$ be an element of the physical mesh $\mathcal{M}$. The next step relates the weak derivatives to the definition (4.4). By the chain rule and the regularity of $\tilde{\boldsymbol{\Phi}}$ we have

$$
\partial_{t} v=\partial_{t}\left[(v \circ \tilde{\boldsymbol{\Phi}}) \circ \tilde{\boldsymbol{\Phi}}^{-1}\right]=\hat{\nabla}(v \circ \tilde{\boldsymbol{\Phi}}) \circ \tilde{\boldsymbol{\Phi}}^{-1} \cdot \partial_{t} \tilde{\boldsymbol{\Phi}}^{-1} .
$$

We denote by $\hat{\nabla}$ the gradient with respect to the coordinates $\zeta_{1}, \ldots, \zeta_{d+1}$ of the parametric domain $\hat{\mathcal{Q}}$. Further rearrangements yield with the structure of $\tilde{\boldsymbol{\Phi}}$ (see (3.1)),

$$
\partial_{t} v=\frac{1}{T} \partial_{\zeta_{d+1}}(v \circ \tilde{\mathbf{\Phi}}) \circ \tilde{\boldsymbol{\Phi}}^{-1}=\frac{1}{T} D_{\tilde{\boldsymbol{\Phi}}}^{(0, \ldots, 0,1)} v .
$$

Moreover, using again the chain rule it holds that

$$
\begin{aligned}
\partial_{x_{i}} \partial_{t} v & =\frac{1}{T} \partial_{x_{i}}\left[\partial_{\zeta_{d+1}}(v \circ \tilde{\mathbf{\Phi}}) \circ \tilde{\boldsymbol{\Phi}}^{-1}\right]=\frac{1}{T} \hat{\nabla}\left[\partial_{\zeta_{d+1}}(v \circ \tilde{\mathbf{\Phi}})\right] \circ \tilde{\boldsymbol{\Phi}}^{-1} \cdot \partial_{x_{i}} \tilde{\boldsymbol{\Phi}}^{-1} \\
& =\frac{1}{T} \sum_{j=1}^{d}\left[\partial_{\zeta_{j}} \partial_{\zeta_{d+1}}(v \circ \tilde{\boldsymbol{\Phi}}) \circ \tilde{\boldsymbol{\Phi}}^{-1}\right]\left(\partial_{x_{i}} \tilde{\boldsymbol{\Phi}}^{-1}\right)_{j} .
\end{aligned}
$$

Let $\boldsymbol{e}_{j} \in \mathbb{N}^{d+1}$ be the $j$-th canonical basis vector. Then we can choose a constant $C=C\left(\tilde{\boldsymbol{\Phi}}^{-1}\right)$ such that

$$
\left|\partial_{x_{i}} \partial_{t} v\right| \leq C \sum_{j=1}^{d}\left|\partial_{\zeta_{j}} \partial_{\zeta_{d+1}}(v \circ \tilde{\boldsymbol{\Phi}}) \circ \tilde{\boldsymbol{\Phi}}^{-1}\right|=C \sum_{j=1}^{d}\left|D_{\tilde{\boldsymbol{\Phi}}}^{\boldsymbol{e}_{j}+(0, \ldots, 0,1)} v\right|
$$


Consequently, (4.5) and (4.6) imply for some (new) constant $C$ only depending on the parametrization that

$$
\begin{gathered}
\left\|\partial_{t} v\right\|_{L^{2}(\mathcal{K})} \leq C\left\|D_{\tilde{\boldsymbol{\Phi}}}^{(0, \ldots, 0,1)} v\right\|_{L^{2}(\mathcal{K})} \\
\left\|\partial_{x_{i}} \partial_{t} v\right\|_{L^{2}(\mathcal{K})} \leq C \sum_{j=1}^{d}\left\|D_{\tilde{\boldsymbol{\Phi}}}^{\boldsymbol{e}_{j}+(0, \ldots, 0,1)} v\right\|_{L^{2}(\mathcal{K})}
\end{gathered}
$$

Similarly one gets

$$
\begin{aligned}
\left\|\partial_{x_{i}} v\right\|_{L^{2}(\mathcal{K})} & \leq C \sum_{j=1}^{d}\left\|D_{\tilde{\mathbf{\Phi}}}^{\boldsymbol{e}_{j}} v\right\|_{L^{2}(\mathcal{K})}, \\
\|v\|_{L^{2}(\mathcal{K})} & =\left\|D_{\tilde{\mathbf{\Phi}}}^{(0, \ldots, 0)} v\right\|_{L^{2}(\mathcal{K})} .
\end{aligned}
$$

Again by the chain rule and an induction argument one gets a reverse estimate, namely

$$
\left\|D_{\tilde{\boldsymbol{\Phi}}}^{\boldsymbol{s}} u\right\|_{L^{2}(\mathcal{K})} \leq C\|v\|_{H^{s}(\mathcal{K})}, \quad \text { for } v \in H^{s}(\mathcal{K}) \text { with } s=s_{1}+\cdots+s_{d+1} .
$$

Observing the regularity of the mesh and that $h_{T}$ and $h_{S}$ denote the mesh sizes in the spatial domain and in the time interval, the assertion follows from the inequalities (4.7)-(4.10) and (4.11) together with [14, Theorem 7 in Part 3]. Therein the proof is only shown in detail for the two-dimensional case, i.e., in our setting for the case $\mathcal{Q} \subset \mathbb{R}^{2}$. But the authors remark the possibility to generalize the proofs and the results straightforwardly also to higher-dimensional spaces. For the case of homogeneous boundary conditions one gets similar estimates due to [14, Remark 14 in Part 3]. This finishes the proof.

REMARK 4.4. In view of the last lemma we can incorporate homogeneous Dirichlet boundary conditions on the whole or on part of the boundary without changing the approximation behavior of the NURBS spaces or NURBS projections, respectively. Thus, we find projections

$$
\begin{gathered}
\Pi_{h}^{\mathcal{W}}: \mathcal{W}_{0} \rightarrow \mathcal{W}_{h, r_{p}, r_{T}}, \\
\Pi_{h}^{\mathcal{V}}: \mathcal{V}_{0} \rightarrow \mathcal{V}_{h, r_{u}, r_{T}},
\end{gathered}
$$

with $\Pi_{h}^{\mathcal{V}}$ acting componentwise, i.e., $\left(\Pi_{h}^{\mathcal{V}} \boldsymbol{u}\right)_{i}:=\Pi_{h}^{\mathcal{V}} u_{i}$, where $\Pi_{h}^{\mathcal{W}}, \Pi_{h}^{\mathcal{V}}$ satisfy the same estimates as $\Pi_{h}$ in the last lemma, potentially with new constants. and $\|\cdot\|_{h, \star} \cdot$

Now we can state an approximation result for the NURBS spaces in the norms $\|\cdot\|_{h}$

LEMMA 4.5. Let $s_{1}, s_{2}, s_{3} \in \mathbb{N}_{\geq 1}, s_{1} \leq r_{T}+1, s_{2} \leq r_{p}+1, s_{3} \leq r_{u}+1$, where $r_{p}$, $r_{u}$ denote the polynomial degrees in the spatial coordinates and $r_{T}$ the polynomial degree in the temporal parameter. Moreover, let $l_{1} \geq \max \left\{s_{1}, s_{2}\right\}$ and $l_{2} \geq \max \left\{s_{1}+1, s_{3}+1\right\}$.

If $p \in \mathcal{W}_{0} \cap H^{l_{1}}(\mathcal{Q})$ and $\boldsymbol{u} \in \mathcal{V}_{0} \cap \boldsymbol{H}^{l_{2}}(\mathcal{Q})$, then it holds that

$$
\begin{aligned}
\|\boldsymbol{\Pi}[\boldsymbol{u}, p]-[\boldsymbol{u}, p]\|_{h, \star} & \leq C_{\boldsymbol{\Pi}, 1}\left(h_{T}^{s_{1}-1}+h_{S}^{s_{2}-1}+h_{T}^{-0.5}\left(h_{S}^{s_{2}}+h_{S}^{s_{3}-1}\right)\right) \mathcal{C}_{l_{1}, l_{2}}(\boldsymbol{u}, p), \\
\|\boldsymbol{\Pi}[\boldsymbol{u}, p]-[\boldsymbol{u}, p]\|_{h} & \leq C_{\boldsymbol{\Pi}, 2}\left(h_{T}^{s_{1}-1}+h_{S}^{s_{2}-1}+h_{S}^{s_{3}-1}\right) \mathcal{C}_{l_{1}, l_{2}}(\boldsymbol{u}, p)
\end{aligned}
$$

where

$$
\mathcal{C}_{l_{1}, l_{2}}(\boldsymbol{u}, p):=\sqrt{\|\boldsymbol{u}\|_{\boldsymbol{H}^{l_{2}(\mathcal{Q})}}^{2}+\|p\|_{H^{l_{1}(\mathcal{Q})}}^{2}}
$$


Here, the constants $C_{\Pi, 1}, C_{\Pi, 2}$ are independent of the mesh sizes $h_{T}, h_{S}$ and the mappings $p$ and $\boldsymbol{u}$ and also independent of $c_{0}$, and we used the notation

$$
\boldsymbol{\Pi}[\boldsymbol{u}, p]-[\boldsymbol{u}, p]:=\left[\boldsymbol{\Pi}_{h}^{\mathcal{V}} \boldsymbol{u}-\boldsymbol{u}, \Pi_{h}^{\mathcal{W}} p-p\right] .
$$

Proof. We prove the statement for the norm $\|\cdot\|_{h}$ at first and consider each term in its definition (3.10) separately. Doing so we use several times the estimates of Lemma 4.3 and Remark 4.4. Besides, we indicate by $C$ a constant which might change at different occurrences but is independent of the mesh sizes $h_{T}, h_{S}$ and the functions $\boldsymbol{u}, p$.

1. The first summand without the prefactor $h_{T}$ gives us with a suitable constant $C$ and Lemma 4.3,

$$
\begin{aligned}
\left\|\partial_{t}\left(\boldsymbol{\Pi}_{h}^{\mathcal{V}} \boldsymbol{u}-\boldsymbol{u}\right)\right\|_{\mathcal{H}^{1}(\mathcal{Q})}^{2} & \leq \sum_{i, j}\left\|\partial_{x_{i}} \partial_{t}\left(\boldsymbol{\Pi}_{h}^{\mathcal{V}} u_{j}-u_{j}\right)\right\|_{L^{2}(\mathcal{Q})}^{2} \\
& \quad+\sum_{i}\left\|\partial_{t}\left(\boldsymbol{\Pi}_{h}^{\mathcal{V}} u_{i}-u_{i}\right)\right\|_{L^{2}(\mathcal{Q})}^{2} \\
& \leq C\left(h_{T}^{s_{1}-1}+h_{S}^{s_{3}-1}\right)^{2}\|\boldsymbol{u}\|_{\boldsymbol{H}^{l_{2}(\mathcal{Q})}}^{2} .
\end{aligned}
$$

2. For the second term we use integration by parts, the chain rule, and Lemma 4.3:

$$
\begin{aligned}
\left\|\boldsymbol{\Pi}_{h}^{\mathcal{V}} \boldsymbol{u}-\boldsymbol{u}\right\|_{\boldsymbol{H}^{1}\left(\Sigma_{T}\right)}^{2} & =\sum_{i}\left\|\boldsymbol{\Pi}_{h}^{\mathcal{V}} u_{i}-u_{i}\right\|_{H^{1}\left(\Sigma_{T}\right)}^{2} \\
= & \sum_{i, j} \int_{\mathcal{Q}} \partial_{t}\left[\left(\partial_{x_{j}}\left(\boldsymbol{\Pi}_{h}^{\mathcal{V}} u_{i}-u_{i}\right)\right)^{2}\right] d \boldsymbol{x} d t \\
& \quad+\sum_{i} \int_{\mathcal{Q}} \partial_{t}\left[\left(\Pi_{h}^{\mathcal{V}} u_{i}-u_{i}\right)^{2}\right] d \boldsymbol{x} d t \\
\leq & C\left\|\partial_{t}\left(\boldsymbol{\Pi}_{h}^{\mathcal{V}} \boldsymbol{u}-\boldsymbol{u}\right)\right\|_{\mathcal{H}^{1}(\mathcal{Q})}\left\|\boldsymbol{\Pi}_{h}^{\mathcal{V}} \boldsymbol{u}-\boldsymbol{u}\right\|_{\mathcal{H}^{1}(\mathcal{Q})} \\
\leq & C\left(h_{T}^{s_{1}-1}+h_{S}^{s_{3}-1}\right)^{2}\|\boldsymbol{u}\|_{\boldsymbol{H}^{l_{2}(\mathcal{Q})}}^{2} .
\end{aligned}
$$

In the second line, we assumed $\partial_{x_{j}} u=0$ on $\Sigma_{0}$ in the sense of the trace theorem. This is indeed correct due to the $\boldsymbol{H}^{2}$-regularity of $\boldsymbol{u}$ and the condition $\boldsymbol{u}=0$ on $\Sigma_{0}$.

3. In an analogous fashion, one has

$$
\left\|\Pi_{h}^{\mathcal{W}} p-p\right\|_{L^{2}\left(\Sigma_{T}\right)}^{2} \leq C\left(h_{T}^{s_{1}-1}+h_{S}^{s_{2}-1}\right)^{2}\|p\|_{H^{l_{1}(\mathcal{Q})}}^{2},
$$

where we again used Lemma 4.3.

4. Furthermore, we obtain with the above lemma that

$$
\begin{aligned}
\left\|\partial_{t}\left(\Pi_{h}^{\mathcal{W}} p-p\right)\right\|_{L^{2}(\mathcal{Q})}^{2}+\left\|\nabla_{x}\left(\Pi_{h}^{\mathcal{W}} p-p\right)\right\|_{L^{2}(\mathcal{Q})}^{2} & \\
& \leq C\left(h_{T}^{s_{1}-1}+h_{S}^{s_{2}-1}\right)^{2}\|p\|_{H^{l_{1}}(\mathcal{Q})}^{2} .
\end{aligned}
$$

The last four estimates yield

$$
\begin{aligned}
&\|\boldsymbol{\Pi}[\boldsymbol{u}, p]-[\boldsymbol{u}, p]\|_{h}^{2} \leq C[(\left(h_{T}\left(h_{T}^{s_{1}-1}+h_{S}^{s_{3}-1}\right)^{2}+\left(h_{T}^{s_{1}-1}+h_{S}^{s_{3}-1}\right)^{2}\right)\|\boldsymbol{u}\|_{\boldsymbol{H}^{l_{2}}(\mathcal{Q})}^{2} \\
&\left.+\left(\left(h_{T}^{s_{1}-1}+h_{S}^{s_{2}-1}\right)^{2}+\left(h_{T}^{s_{1}-1}+h_{S}^{s_{2}-1}\right)^{2}\right)\|p\|_{H^{l_{1}}(\mathcal{Q})}^{2}\right] \\
& \leq C\left(h_{T}^{s_{1}-1}+h_{S}^{s_{2}-1}+h_{S}^{s_{3}-1}\right)^{2}\left(\|\boldsymbol{u}\|_{\boldsymbol{H}^{l_{2}(\mathcal{Q})}}^{2}+\|p\|_{H^{l_{1}}(\mathcal{Q})}^{2}\right) .
\end{aligned}
$$


This implies (4.15). One notes the assumption $c_{0} \leq 1$.

For the $\|\cdot\|_{h, \star}$-norm it is sufficient to consider the parenthesized term in (4.3), which gives with a prefactor, using again Lemma 4.3 and Remark 4.4,

$$
\begin{aligned}
\frac{1}{h_{T}}\left\|\boldsymbol{\Pi}_{h}^{\mathcal{V}} \boldsymbol{u}-\boldsymbol{u}\right\|_{\mathcal{H}^{1}(\mathcal{Q})}^{2}+\frac{1}{h_{T}}\left\|\Pi_{h}^{\mathcal{W}} p-p\right\|_{L^{2}(\mathcal{Q})}^{2} \\
\quad \leq C \frac{1}{h_{T}}\left(h_{T}^{s_{1}}+h_{S}^{s_{3}-1}\right)^{2}\|\boldsymbol{u}\|_{\boldsymbol{H}^{l_{2}(\mathcal{Q})}}^{2}+C \frac{1}{h_{T}}\left(h_{T}^{s_{1}}+h_{S}^{s_{2}}\right)^{2}\|p\|_{H^{l_{1}(\mathcal{Q})}}^{2} \\
\leq C \frac{1}{h_{T}}\left(h_{T}^{s_{1}}+h_{S}^{s_{2}}+h_{S}^{s_{3}-1}\right)^{2}\left(\|\boldsymbol{u}\|_{\boldsymbol{H}^{l_{2}(\mathcal{Q})}}^{2}+\|p\|_{H^{l_{1}}(\mathcal{Q})}^{2}\right) .
\end{aligned}
$$

The last three lines and the already shown inequalities give us the desired bound (4.14) for the norm $\|\cdot\|_{h, \star}$.

Finally, we are ready to prove a convergence estimate for the space-time method.

THEOREM 4.6 (Convergence for smooth solutions). Let the assumptions of Lemma 4.5 be fulfilled, where $l_{2} \geq 3, l_{1} \geq 2$. Moreover let $\boldsymbol{u} \in \mathcal{V}_{0} \cap \boldsymbol{H}^{l_{2}}(\mathcal{Q}), p \in \mathcal{W}_{0} \cap H^{l_{1}}(\mathcal{Q})$ be the exact solution of the Biot system (2.3)-(2.4) with initial-boundary conditions (2.5)-(2.8). Then we have for the error between $\boldsymbol{u}, p$ and the solution $\boldsymbol{u}_{h}, p_{h}$ of the finite-dimensional variational problem (3.8) that

$$
\left\|[\boldsymbol{u}, p]-\left[\boldsymbol{u}_{h}, p_{h}\right]\right\|_{h} \leq C\left(h_{T}^{s_{1}-1}+h_{S}^{s_{2}-1}+h_{T}^{-0.5}\left(h_{S}^{s_{2}}+h_{S}^{s_{3}-1}\right)\right) \mathcal{C}_{l_{1}, l_{2}}(\boldsymbol{u}, p),
$$

where $C$ is some constant that does not depend on $c_{0}$ and the mesh sizes $h_{T}, h_{S}$.

Proof. We make use of the coercivity and continuity of $b_{S T}$ shown in Lemma 3.8 and Lemma 4.2 and obtain with the NURBS projections

$$
\boldsymbol{\Pi}[\boldsymbol{u}, p]:=\left[\Pi_{h}^{\mathcal{V}} \boldsymbol{u}, \Pi_{h}^{\mathcal{W}} p\right], \quad \Pi_{h}^{\mathcal{W}}: \mathcal{W}_{0} \rightarrow \mathcal{W}_{h, r_{p}, r_{T}} \quad \text { and } \quad \Pi_{h}^{\mathcal{V}}: \mathcal{V}_{0} \rightarrow \mathcal{V}_{h, r_{u}, r_{T}}
$$

that

$$
\begin{aligned}
\mu_{c}\left\|\boldsymbol{\Pi}[\boldsymbol{u}, p]-\left[\boldsymbol{u}_{h}, p_{h}\right]\right\|_{h}^{2} & \leq b_{S T}\left(\boldsymbol{\Pi}[\boldsymbol{u}, p]-\left[\boldsymbol{u}_{h}, p_{h}\right], \boldsymbol{\Pi}[\boldsymbol{u}, p]-\left[\boldsymbol{u}_{h}, p_{h}\right]\right) \\
& =b_{S T}\left(\boldsymbol{\Pi}[\boldsymbol{u}, p]-[\boldsymbol{u}, p], \boldsymbol{\Pi}[\boldsymbol{u}, p]-\left[\boldsymbol{u}_{h}, p_{h}\right]\right) \\
& \leq \mu_{b}\|\boldsymbol{\Pi}[\boldsymbol{u}, p]-[\boldsymbol{u}, p]\|_{h, \star}\left\|\boldsymbol{\Pi}[\boldsymbol{u}, p]-\left[\boldsymbol{u}_{h}, p_{h}\right]\right\|_{h}
\end{aligned}
$$

Note that we used the consistency result of Lemma 3.4. The above inequality chain implies

$$
\left\|\boldsymbol{\Pi}[\boldsymbol{u}, p]-\left[\boldsymbol{u}_{h}, p_{h}\right]\right\|_{h} \leq \frac{\mu_{b}}{\mu_{c}}\|\boldsymbol{\Pi}[\boldsymbol{u}, p]-[\boldsymbol{u}, p]\|_{h, \star} .
$$

We remark for the above inequality that $\boldsymbol{\Pi}[\boldsymbol{u}, p]-\left[\boldsymbol{u}_{h}, p_{h}\right] \in \mathcal{V}_{h, r_{u}, r_{T}} \times \mathcal{W}_{h, r_{p}, r_{T}}$. By the previous inequality and Lemma 4.5 it follows that

$$
\begin{aligned}
\left\|[\boldsymbol{u}, p]-\left[\boldsymbol{u}_{h}, p_{h}\right]\right\|_{h} & \leq\left\|\boldsymbol{\Pi}[\boldsymbol{u}, p]-\left[\boldsymbol{u}_{h}, p_{h}\right]\right\|_{h}+\|\boldsymbol{\Pi}[\boldsymbol{u}, p]-[\boldsymbol{u}, p]\|_{h} \\
& \leq \frac{\mu_{b}}{\mu_{c}}\|\boldsymbol{\Pi}[\boldsymbol{u}, p]-[\boldsymbol{u}, p]\|_{h, \star}+\|\boldsymbol{\Pi}[\boldsymbol{u}, p]-[\boldsymbol{u}, p]\|_{h} \\
& \leq \frac{\mu_{b}}{\mu_{c}} C_{\boldsymbol{\Pi}, 1}\left(h_{T}^{s_{1}-1}+h_{S}^{s_{2}-1}+h_{T}^{-0.5}\left(h_{S}^{s_{2}}+h_{S}^{s_{3}-1}\right)\right) \mathcal{C}_{l_{1}, l_{2}}(\boldsymbol{u}, p) \\
& \quad+C_{\boldsymbol{\Pi}, 2}\left(h_{T}^{s_{1}-1}+h_{S}^{s_{2}-1}+h_{S}^{s_{3}-1}\right) \mathcal{C}_{l_{1}, l_{2}}(\boldsymbol{u}, p) \\
& \leq C\left(h_{T}^{s_{1}-1}+h_{S}^{s_{2}-1}+h_{T}^{-0.5}\left(h_{S}^{s_{2}}+h_{S}^{s_{3}-1}\right)\right) \mathcal{C}_{l_{1}, l_{2}}(\boldsymbol{u}, p),
\end{aligned}
$$

for some constant $C$, e.g., $C=\left(\frac{\mu_{b}}{\mu_{c}} C_{\Pi, 1}+C_{\Pi, 2}\right)$ if $h_{T} \leq 1$. 
How does this convergence result compare with the vertical method of lines? In the latter case, the spatial discretization leads to an ODE or DAE in time which is then solved by specific integration schemes. In Theorem 4.6, the spatial and temporal influences show up in terms of $h_{S}^{a}, h_{T}^{a}$, respectively. If we assume $h_{S}$ being of order $\mathcal{O}\left(h_{T}\right)$ and require a regular exact solution, then the error in the norm $\|\cdot\|_{h}$ is of order

$$
\mathcal{O}\left(h_{T}^{r_{T}}+h_{S}^{r_{p}}+h_{T}^{-0.5}\left(h_{S}^{r_{p}+1}+h_{S}^{r_{u}}\right)\right)=\mathcal{O}\left(h_{T}^{r_{T}}+h_{S}^{r_{p}}+h_{S}^{r_{u}-0.5}\right) .
$$

Error estimates for the method of lines typically have the form error $\leq$ const. $\cdot\left(\Delta t^{m}+h_{S}^{k}\right)$, where $m$ denotes the convergence order of the time integrator, $\Delta t$ the step size, and $k$ the approximation order of the spatial discretization.

From a theoretical point of view it is possible to obtain high orders of convergence in both ways, but for the space-time method, increasing the convergence order can be achieved by raising the polynomial degrees, which becomes very efficient in the context of IGA. Variable-order multistep formulae also allow one to do this, in particular the BDF method, while for implicit and linear-implicit one-step methods such as the Runge-Kutta scheme, the order is fixed. Besides, for multistep methods the choice of good starting values is of relevance, and order reduction problems might occur for Runge-Kutta-type algorithms; compare [18, Remark 6 and 7].

The case of the continuous P1-B-spline discretization in time within the space-time method deserves a further remark. For illustration, we apply the method based on the timeupwind test functions introduced in [26], which is similar to our discretization of the Biot system, to the semi-discretized homogeneous heat equation $\boldsymbol{M}_{h} \partial_{t} \boldsymbol{w}=\boldsymbol{A}_{h} \boldsymbol{w}$. Here we have an spd mass matrix $\boldsymbol{M}_{h}$ and a semi-definite right-hand side matrix $\boldsymbol{A}_{h}$. More precisely, we make the ansatz $\boldsymbol{w}=\sum_{k} \boldsymbol{w}_{k} \phi_{k}(t)$, where $\phi_{k}$ are P1-B-splines, and multiply the system by test functions $\left(\phi_{k}(t)+\Delta t \partial_{t} \phi_{k}(t)\right) \boldsymbol{e}_{i}$, where $\boldsymbol{e}_{i}$ denotes the $i$-th canonical basis vector, and integrate with respect to time. Instead of a simultaneous space-time discretization, we split the procedure thus into two discretization steps and obtain, after some straightforward computations, the recursion formula

$$
\boldsymbol{M}_{h}\left(-\frac{3}{4} \boldsymbol{w}_{k-1}+\boldsymbol{w}_{k}-\frac{1}{4} \boldsymbol{w}_{k+1}\right)=\Delta t \boldsymbol{A}_{h}\left(\frac{1}{3} \boldsymbol{w}_{k-1}+\frac{1}{3} \boldsymbol{w}_{k}-\frac{1}{6} \boldsymbol{w}_{k+1}\right)
$$

for the time steps $k=1,2, \ldots$ We observe the structure of an implicit 2-step method, and the consistency order is readily verified to be $m=3$. The difference to a classical multistep approach that would proceed step by step lies in the first step $k=1$, where both $\boldsymbol{w}_{1}$ and $\boldsymbol{w}_{2}$ are unknowns as there is no starting procedure for $\boldsymbol{w}_{1}$. If we arrange all time steps in a large linear system, then it has a tridiagonal staircase block structure that reflects the propagation of information. Thus, the P1-B-spline temporal discretization is inappropriate for a simple sequential processing in time. The same reasoning applies to the full space-time method for the Biot system. It is thus natural to treat the fully discretized system en bloque by suitable sparse direct or iterative methods that in the end take implicitly advantage of the staircase structure.

Finally, the factor $h_{T}^{-0.5}$ in the estimate (4.17) suggests an order reduction effect. The appearance of this factor is due to the use of the norm $\|\cdot\|_{h, \star}$ in the boundedness estimate for $b_{S T}$ (see Lemma 4.2), where in the defining equation (4.3) a factor $1 / h_{T}$ appears. Our numerical experiments below, however, did not reveal this order reduction, which indicates that our estimate might be too pessimistic. For clarification, we come back to the issue of the norm $\|\cdot\|_{h, \star}$ in the following numerics part.

5. Numerical examples. In this section we focus on numerical examples for validating the convergence behaviour, but also instability issues are considered. The numerical 
experiments are performed by means of the powerful GeoPDEs package [16, 39], which is a MATLAB [29] implementation of IGA. We write IG-ST as an abbreviation for the space-time method introduced above. Further, the following test examples have also been computed by means of a method-of-lines ansatz, namely a spatial isogeometric discretization combined with a BDF time-stepping. The obtained results are not documented here but were used to verify the plausibility of the IG-ST solutions. The overall linear system of the space-time method is solved by MATLAB's sparse direct solver. This means that we concentrate here on verifying convergence and treat the linear algebra as a black box. Of course, in this regard, there is room for substantial adaptation and tuning.

5.1. Numerical convergence analysis. By manufacturing the right-hand sides, we construct the smooth strong solution

$$
\begin{aligned}
u_{1}(\boldsymbol{x}, t) & =\sin (\pi x) \sin (\pi y) \sin (\pi t), & u_{2}(\boldsymbol{x}, t)=\sin (\pi x) \sin (\pi y)(\exp (t)-1), \\
p(\boldsymbol{x}, t) & =\sin (\pi x) \sin (\pi y) \sin (0.5 \pi t), & (x, y):=\boldsymbol{x},
\end{aligned}
$$

of the Biot system, where the spatial domain is the unit square and the time interval is $[0, T]=[0,1]$. Hence, the space-time cylinder $\mathcal{Q}$ is the three-dimensional unit cube. Uniform meshes $\mathcal{M}_{h}$ are obtained by dividing the cube into equally smaller cubes with edge lengths $h=2^{-k}, k=1, \ldots, 5$. In other words we ignore here the difference between spatial and temporal mesh sizes and have $h=h_{T}=h_{S}$. We assume homogeneous initial-boundary conditions on the surface of the space-time cylinder. For the coefficients and parameters, respectively, we set $c_{0}=1, \lambda=1, \mu=1, \mathcal{K}=\boldsymbol{I}, b=1$. For the underlying discrete NURBS spaces we use simple inner knots, and in order to save computational costs, we apply basis functions that are also $r_{T}-1$-times continuously differentiable in time.

First we choose a mixed ansatz in space for the polynomial degrees, namely $r_{p}=r_{T}$ and $r_{u}=r_{p}+1$. Due to the regularity of the strong solution and the choice of the boundary conditions, we expect the error in the norm $\|\cdot\|_{h}$ to be of the order $\mathcal{O}\left(h^{r_{p}}+h^{r_{u}-0.5}\right)$; see Theorem 4.6. We compute the errors in the $\|\cdot\|_{h}$-norm for the different meshes $\mathcal{M}_{h}$, $h=1 / 2, \ldots, 1 / 32$, and the polynomial degrees $r_{p}=1,2,3$. The result is presented in Figure 5.1(a).

Additionally we display in Figure 5.1(b) the computed $\|\cdot\|_{h}$-norm errors but with equal degrees $r_{p}=r_{T}=r_{u}$, for $r_{p}=1,2,3$. One observes a similar convergence behaviour as for the mixed-degrees case although our convergence estimate indicates an order reduction. Thus, one might conjecture that the convergence estimates are not yet optimal. As mentioned before, the factor $h_{T}^{-0.5}$ in the error estimate of Theorem 4.6 arises from the definition of the norm $\|\cdot\|_{h, \star}$, which is needed to state a boundedness inequality for $b_{S T}$. To highlight the influence of $h_{T}$, we repeat the above convergence example with a fixed spatial mesh size; more precisely, we set $h_{S}=0.25$, but we decrease $h_{T}=2^{-k}$ with $k=1, \ldots, 8$. The errors in both norms $\|\cdot\|_{h}$ and $\|\cdot\|_{h, \star}$ are displayed in Figure 5.2(a)-(b). Indeed we can observe an error increase of approximately of order $\mathcal{O}\left(h_{T}^{-0.5}\right)$ with respect to the $\star$-norm, whereas the errors in the $\|\cdot\|_{h}$-norm stagnate. To demonstrate the convergence behaviour for the extreme case $c_{0}=0$, we display in Figure 5.1(c)-(d) the $\|\cdot\|_{h}$-norm errors for the above test problem with $c_{0}$ set to zero.

5.2. Terzaghi's problem and the problem of Barry and Mercer. Next, we give two examples from the literature for which an analytical solution is known. Terzaghi's problem is a one-dimensional model with an analytical pressure solution that describes the coupling of the fluid pressure and the deformation of a porous medium pipe completely filled with some fluid if one end is fixed and at the other end a uniform normal surface load $\boldsymbol{F}=(F, 0)$ is applied; see Figure 5.3. We require the displacement and the fluid flow to be restricted parallel 




(a) $r_{p}=r_{T}, r_{u}=r_{p}+1$, and $c_{0}=1$.



(c) $r_{p}=r_{T}, r_{u}=r_{p}+1$, and $c_{0}=0$.



(b) $r_{p}=r_{T}=r_{u}$ and $c_{0}=1$.



(d) $r_{p}=r_{T}=r_{u}$ and $c_{0}=0$.

FIG. 5.1. Errors in the norm $\|\cdot\|_{h}$ for mixed and non-mixed polynomial degrees as well as for the two cases $c_{0}=1$ and $c_{0}=0$.

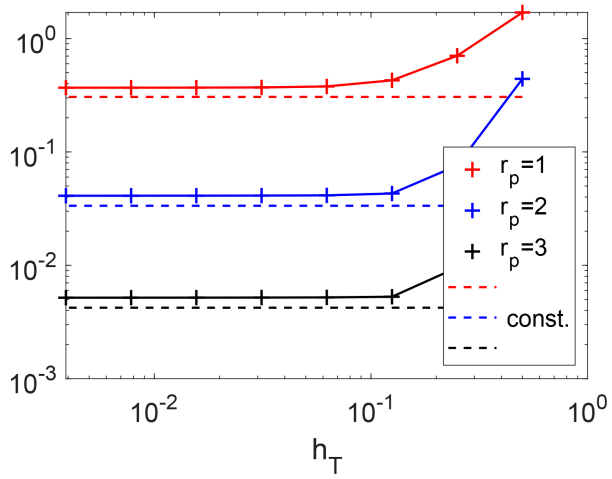

(a) The $\|\cdot\|_{h}$-errors for fixed $h_{S}, r_{p}=r_{T}$, $r_{u}=r_{p}+1$, and $c_{0}=1$.



(b) The $\|\cdot\|_{h, \star}$-errors for fixed $h_{S}, r_{p}=r_{T}$, $r_{u}=r_{p}+1$, and $c_{0}=1$.

FIG. 5.2. Errors for mixed polynomial degrees and fixed spatial mesh size $h_{S}=1 / 4$. 
to the pipe such that the problem can be reduced to a one-dimensional setting. The governing equations are given by the $1 \mathrm{D}$ Biot system, where the physical domain $\Omega$ reduces to some interval $\Omega=(0, L)$ and the permeability tensor is given by some positive constant $k$. The



FIG. 5.3. Terzaghi's problem: Saturated porous medium deformed by some loading.

boundary and initial conditions are set to

$$
\begin{aligned}
p=0, \boldsymbol{t}_{n}=F, 0<F<\infty & \text { at } x=0, \\
\partial_{x} p=0, u=0 & \text { at } x=L, 0<L<\infty, \\
p(t=0)=u(t=0)=0 . &
\end{aligned}
$$

The exact pressure solution can be found in [31]. We set the parameters to $c_{0}=0.2$, $\lambda=1, \mu=1, k=0.2, b=1, \eta_{f}=1$, and $F=L=1$, and the space-time cylinder is $\mathcal{Q}=(0,1) \times(0,2)$. We use a uniform mesh with mesh sizes $h_{T}=0.01, h_{S}=0.05$ and polynomial degree 1 in each coordinate. In Figure 5.4(a), the numerical solution is displayed. The approximate values fit the exact solution quite well. One observes that the deviation is at a maximum for $t=0.05$, which can be explained by the following reasoning: The exact solution converges for $t \rightarrow 0$ pointwisely to some discontinuous function $x \mapsto p_{0} \chi_{(0,1]}(x)$, where $\chi$ is the indicator function. However, the IG-ST method is used with zero initial conditions and yields only globally continuous solutions. Thus, the non-smooth limit behavior can not be reproduced by the IG-ST method, and the deviation between exact and numerical solution grows for $t \rightarrow 0$ as exemplified in Figure 5.4(b) for $t=0.01$. This drawback can be alleviated



(a) Results for $h_{T}=0.01, h_{S}=0.05$.



(b) Early time solution at $t=0.01$.

FIG. 5.4. Comparison between the exact solution of the Terzaghi test problem and the approximate solutions. The deviation grows for $t \rightarrow 0$.

by using finer meshes. At first glance, degree elevation for the time parameter seems to be another way for improving the solution at early times. But numerical tests not shown here 




FIG. 5.5. Boundary conditions for the problem of Barry and Mercer. Above, the dotted lines and the highlighted square indicate the spatial mesh for the case $h_{S}=1 / 6$ and the square $S_{h_{S}}$ for the point source approximation, respectively.

indicate that p-refinement in time without mesh refinement essential does not lead to better results since we still need continuity in time for our numerical method, which does not fit to the mentioned limit behavior of the exact solution as $t \rightarrow 0$.

We proceed with a two-dimensional test problem, the problem of Barry and Mercer, which is taken from [18]. This problem describes the pressure and displacement in a rectangular porous medium under the influence of an oscillating fluid point source. Though it has an analytical solution, it is only available in the form of an infinite double series. Moreover, the source term is actually given by a distribution and not by a function. This implies the necessity to approximate the source term by a proper function. Clearly, this test problem does not really fit into the mathematical framework needed for the establishment of the error estimates and the existence statements in the previous sections of the article. Nevertheless, since the number of non-manufactured test cases for the Biot system with analytical solution is small, the Barry and Mercer problem is often addressed in the literature as a test to study different numerical methods. Here we focus on the plausibility of the approximate solution rather than on a convergence study.

More precisely, we have the setting $\Omega=(0,1)^{2}$, and the only source distribution is $g(\boldsymbol{x}, t)=2 \beta \delta\left(\boldsymbol{x}-\boldsymbol{x}_{0}\right) \sin (\beta t)$, where $\delta\left(\boldsymbol{x}-\boldsymbol{x}_{0}\right)$ denotes the Dirac delta distribution at $\boldsymbol{x}_{\mathbf{0}}:=\left(x_{0}, y_{0}\right)$ and $\beta=(\lambda+2 \mu) k$. Further, we use homogeneous Dirichlet boundary conditions for the pressure variable and a mixture of homogeneous Neumann and Dirichlet boundary conditions for the displacement variables; see Figure 5.5. The analytical solution of the problem is stated in [31, Section 4.2.1]. The corresponding parameters taken from [18] are $c_{0}=0, \lambda=10^{4} / 0.88, \mu=10^{5} / 2.2, \mathcal{K}=k \cdot \boldsymbol{I}, k=0.01, b=1$, and $\boldsymbol{x}_{0}=(0.25,0.25)$. Note that the approximation of the Dirac delta is realized in the following way: We partition $\Omega$ into equal squares by means of the spatial IGA mesh, and for the edge length $h_{S}$ of the squares, we denote with $S_{h_{S}}$ the mesh element for which it holds that $\boldsymbol{x}_{0} \in S_{h_{S}}$. In Figure 5.5 we illustrate such a partition indicated by dotted lines for the case $h_{S}=1 / 6$. Using this, we approximate $\delta\left(\boldsymbol{x}-\boldsymbol{x}_{0}\right)$ by $\delta_{h}\left(\boldsymbol{x}-\boldsymbol{x}_{0}\right)=h_{S}^{-2}$ if $\boldsymbol{x} \in S_{h_{S}}$ and $\delta_{h}\left(\boldsymbol{x}-\boldsymbol{x}_{0}\right)=0$ otherwise. As spatial mesh sizes we consider $h_{S}=1 / 34$ and $h_{S}=1 / 66$, i.e., a coarser and a finer mesh. The underlying polynomial degrees are one. Then we choose as space-time cylinder $\mathcal{Q}=(0,1)^{2} \times\left(0, \frac{3 \pi}{2 \beta}\right)$ with mesh size in time being $h_{T}=\frac{3 \pi}{36 \beta}$. One notes that the spatial mesh size is much larger than the temporal mesh size. Hence, the distinction between spatial and temporal step size for the space-time variational formulation seems to be reasonable. 




(a) Pressure at $t=0.5 \pi / \beta$.



(c) $x$-displacement at $t=0.5 \pi / \beta$.



(b) Pressure at $t=1.5 \pi / \beta$.

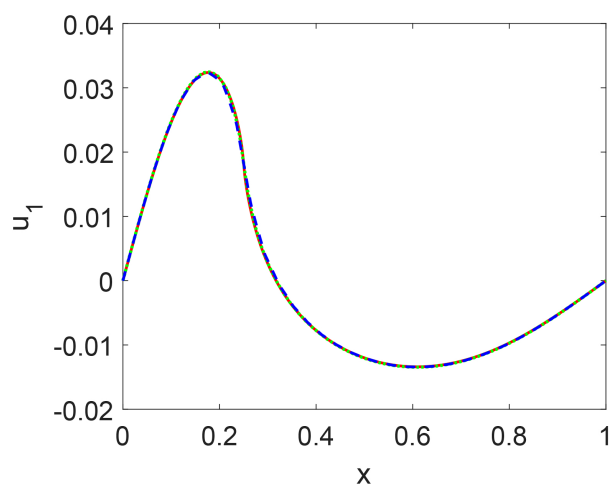

(d) $x$-displacement at $t=1.5 \pi / \beta$.

FIG. 5.6. Comparison between the exact solution and the numerical solutions along the diagonal line $\gamma$ from $(0,0)$ to $(1,1)$.

We compare the numerical solution with a reference solution ${ }^{1}$, which is actually very close to the analytical one and hence suitable for comparisons, and we refer to it as exact solution. For reasons of comparability we plot the exact and numerical solutions of the pressure $p$ and displacement $u_{1}$ along the diagonal line $(0,0)-(1,1)$ (see $\gamma$ in Figure 5.5) of the domain at the times $t_{1}=0.5 \pi / \beta$ and $t_{2}=1.5 \pi / \beta$ in Figure 5.6. The approximate and reference solutions of the displacement in $x$-direction match quite well. The pressure solution deviates near the point source $\boldsymbol{x}_{0}$ due to the coarseness of the mesh and the related approximation of the Dirac delta. For a finer spatial mesh, the results are clearly better near $\boldsymbol{x}_{0}$. We can further exploit the possibility of higher polynomial degrees to achieve better results. For example in Figure 5.7(a)-(b), we illustrate again the numerical pressure and the $x$-displacement solution along the diagonal $\gamma$ at $t_{2}$ but with $h_{S}=1 / 14$ and linear degrees, as well as for $r_{p}=r_{u}=3$, $r_{T}=1$. One can see a clear improvement for the higher degrees. If we increase the degree in the time parameter, i.e., we set $r_{T}=3$ and keep $r_{u}=r_{p}=1$, then we observe improvements especially for small times $t$ as Figure 5.7(c)-(d) demonstrate.

5.3. A 3D geometry with curved boundary. Next, we demonstrate that our space-time method also works for 3D domains with curved boundary, which underlines the advantages

\footnotetext{
${ }^{1}$ The reference solution is computed by means of the analytical solution formula in [31, Section 4.2.1], where we take all summands into account for which both running indices are equal or smaller than 1500 .
} 




(a) Pressure at $t=1.5 \pi / \beta$.

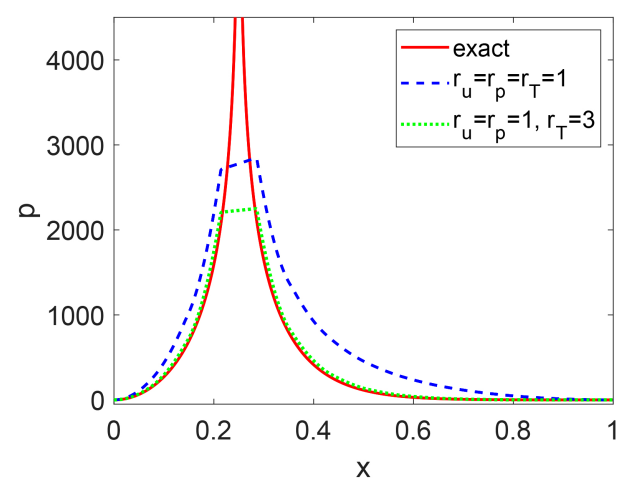

(c) Pressure at $t=0.015 \pi / \beta$.



(b) $x$-displacement at $t=1.5 \pi / \beta$.



(d) $x$-displacement at $t=0.015 \pi / \beta$.

FIG. 5.7. We observe improvements for higher polynomial degrees. In the upper row we illustrate the change if the spatial degree is increased from 1 to 3 . In the bottom row we increase the time parameter degree and display the solution for a small time $t$. Above it is set to $h_{S}=1 / 14$.

of an IGA approach. One notes the fact that for the 3D case, the space-time cylinder is a four-dimensional object. The crescent-shaped geometry with spatial mesh in Figure 5.8(a) is inspired by the porous structure of a human meniscus. From a biomedical viewpoint, the poor vascularization of the meniscus is one reason for premature osteoarthritis in knee joints. On the other hand, the meniscus tissue is highly hydrated (70-75\% water), and the frequent pressure changes during walking and running are essential for the flow of nutrients and for fostering the regeneration capabilities. We prescribe the following parameter values to approximate the behaviour of such a fibro-cartilaginous material: $c_{0}=2.7 \cdot 10^{-10}, \lambda=472689, \mu=183824$, $\mathcal{K}=1.5 \cdot 10^{-12} \cdot \boldsymbol{I}, b=1$. The method parameters are $r_{p}=r_{u}=2, r_{T}=1$ and $h_{T}=1 / 18$, $T=0.5$. As boundary conditions we set the pressure to be zero on the whole boundary except for the flat bottom part of the meniscus, which can move in horizontal directions but is fixed with respect to vertical movements. Both ends of the C-shaped domain are fixed too, and a loading

$$
\boldsymbol{\sigma} \cdot \boldsymbol{n}_{x}=f(t, z)\left(\frac{x}{\sqrt{x^{2}+y^{2}}}, \frac{y}{\sqrt{x^{2}+y^{2}}},-1\right)^{t}
$$

with $f(t, z)=30000 \sin (\pi t) \sin ((1 / 0.0072) \pi z)$ is applied onto the upper surface. 




(a) Spatial mesh of the 3D meniscus model.



(b) Numerical pressure inside the deformed domain at time $t=0.5$.

FIG. 5.8. Mesh and numerical pressure for the case of a meniscus-type domain with curved boundaries.

Figure 5.8 displays the isogeometric mesh and a snapshot of the pressure distribution inside the fibro-cartilaginous material. Here we want to remark that for our used $3 D$ mesh, the problem of a singular Jacobian on two curves on the boundary occurs. In fact, these problematic points are located near the outer radius of the meniscus shape, but due to our relative coarse mesh and the fact that for the numerical computations we only need the Jacobian entries at interior quadrature points, the linear system is still solvable. Presumably, additional stabilization or a different mesh structure is needed for smaller mesh sizes.

5.4. Pressure oscillations and elastic locking. A major issue in solving Biot's equations is the occurrence of spurious pressure oscillations, mainly for low permeability, i.e., if $\|\mathcal{K}\|<<1$. To simplify the discussion, we set $\mathcal{K}=k \boldsymbol{I}$ with a constant $k$. Our numerical experiments show that especially small constrained specific storage coefficients along with low permeability may lead to a nonphysical behaviour. To illustrate this, in Figure 5.9 we plot the approximate solutions to Terzaghi's problem for small parameters $c_{0}=10^{-7}, k=10^{-7}$. As a result, one observes oscillations despite the relatively fine spatial mesh $\left(h_{S}=0.025\right)$ for equal polynomial degrees $r_{p}=r_{u}=1$. These pressure oscillations are well-known and can be handled by an additional stabilization or discontinuous finite element methods. There is also a connection to the locking effect in elasticity [13, Chpt. 6]. In the context of poroelasticity, the pressure variable is more critical, but volumetric locking, i.e., the blocking of the displacements in regions of low-compressible media, can be detected for the Biot system too. Although we can not present a profound theoretical explanation for the instability issue, we want to remark the dependence of the constant $C$ on the permeability in the estimate of Theorem 4.6. More precisely, in view of the coercivity estimate in Lemma 3.8, we can see that for $\|\mathcal{K}\| \rightarrow 0$, the value $\mu_{c}$ tends to zero, an thus, the mentioned constant in Theorem 4.6 blows up to infinity. We see in the latter blow-up a hint for problems in the nearly impermeable case.

The pressure variable can be stabilized by means of a mixed ansatz [7, 21]. For standard IGA combined with an implicit Euler method in time, one can show (using [5, Theorem 5.2.]) that Taylor-Hood mixed spaces satisfy a Babuška-Brezzi inf-sup condition. We follow this 




FIG. 5.9. The Terzaghi problem with small permeability and storativity leads to massive oscillations in the equal-degree case.



FIG. 5.10. Boundary conditions and parameters for the low-permeable layer. For the second test case, i.e., the low-compressible layer, the layer parameters are changed to $\lambda=10^{8}$ and $k=1$.

idea of mixed spaces and increase the polynomial degree in the displacement variable by one. In Figure 5.9 we display the result of the above Terzaghi test case for $r_{u}=r_{p}+1$ in comparison with equal polynomial degrees. The Taylor-Hood ansatz results in an overshooting numerical solution, but it approximates the exact solution quite precisely away from the problematic boundary point $x=0$. Other numerical tests further show that using equal but higher polynomial degrees for both pressure and displacement is not really leading to a substantial improvement. Hence, a significant reduction of the oscillations without the need of very small mesh sizes can only be achieved by a mixed ansatz.

We further illustrate the effect of a mixed ansatz also for the displacement variable by solving two test problems from [21]. In both cases the spatial domain is $\Omega=(0,1)^{2}$. At first, we place a low-permeable layer inside a material with moderate parameters, and for the second case, we place there a low-compressible layer. More precisely, in the first case we have a region in which $k<<1$ is very small, and in the other case we analogously have a large Lamé coefficient $\lambda>>\mu=1$. We set $c_{0}=0, \mu=1, b=1$; see also Figure 5.10. For the case with a low-compressible layer, we change the parameter in the layer from $\lambda=1$, $k=10^{-8}$ to $\lambda=10^{8}, k=1$.

The first test case verifies the reduction of pressure oscillations, and the second one analyzes the elastic locking effect. On the top edge of the domain we apply a constant non-uniform normal load, namely

$$
\boldsymbol{\sigma} \cdot \boldsymbol{n}_{x}=\mathbf{0} \quad \text { for } x<0.5 \quad \text { and } \quad \boldsymbol{\sigma} \cdot \boldsymbol{n}_{x}=-\boldsymbol{n}_{x} \quad \text { for } x \geq 0.5 .
$$

The computed solutions at $t=1$ for zero initial conditions and the two scenarios $1=r_{p}=r_{u}$ and $2=r_{p}+1=r_{u}$ are summarized in Figure 5.11. Here we used a relatively fine uniform mesh with spatial mesh size $h_{S}=1 / 40$ as well as $h_{T}=0.2, r_{T}=1$, and we plott the solution along the line $(0.75,0)-(0.75,1)$. As already observed for Terzaghi's problem, we get a better result for the pressure solution and the low-permeable layer if we use mixed polynomial degrees. The displacement is similar for the mentioned layer. A look at the low-compressible layer case verifies the smoothness of the pressure solution and the absence of oscillations. But the displacement variables differ for both cases. For degrees $r_{p}=r_{u}=1$, the displacement in the layer region is nearly constant. Consequently, the displacement is locked, and we have the presence of elastic locking. However, for the case of choosing $r_{u}=r_{p}+1=2$, the vertical displacement is more plausible. Thus, the locking phenomenon is also damped for 


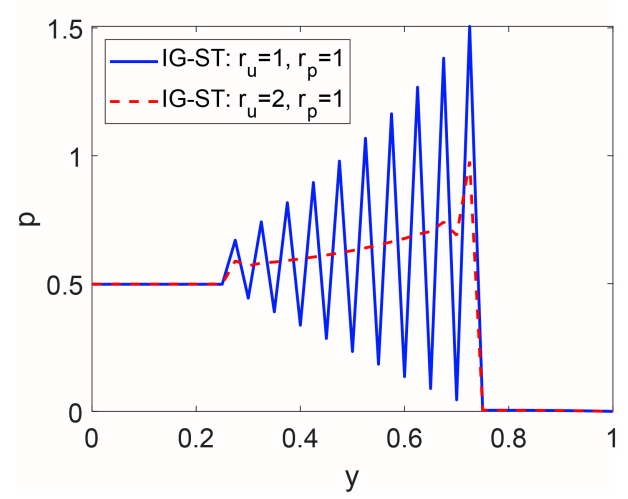

(a) Pressure for low-permeable layer.

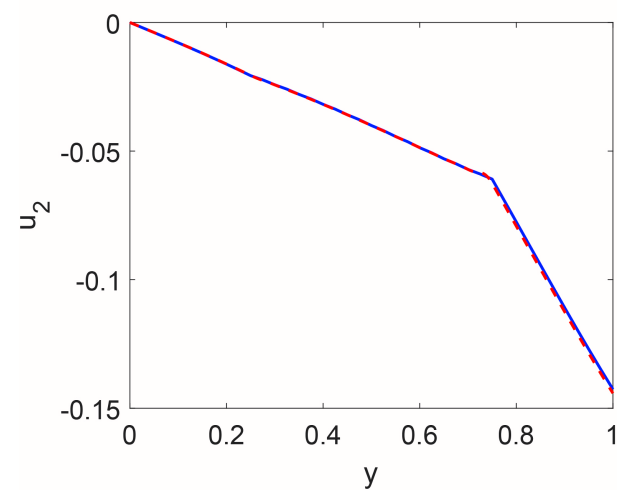

(c) $y$-displacement for low-permeable layer.

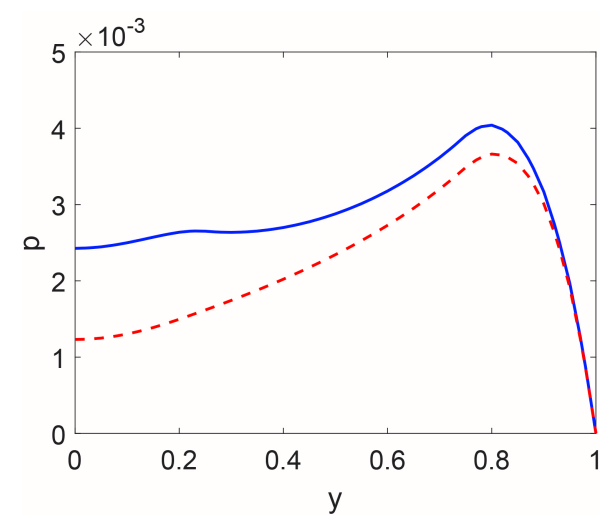

(b) Pressure for low-compressible layer.

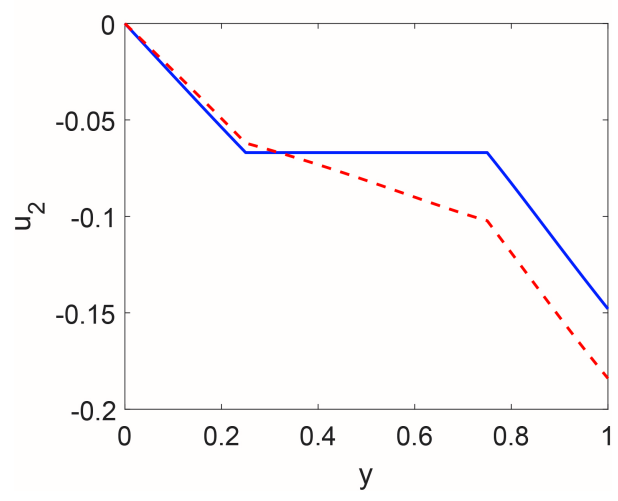

(d) $y$-displacement for low-compressible layer.

FIG. 5.11. The results for the IG-ST method and the test cases of low-permeable and a low-compressible layer along the plot line $(0.75,0)-(0.75,1)$ at the final time $T=1$. Numerical solutions for mixed degrees are plotted in red and for equal degrees in blue, respectively. Mixed degrees reduce the pressure oscillations and the locking effect.

mixed degrees. Another possibility of adapting the spaces for more stability is to play with the smoothness of the test functions spaces, especially for the displacement variable. Indeed, in experiments we observe a similar behavior of the numerical solutions for the latter two stability test examples in case of an increased $C^{1}$-continuity for the displacement test functions if $r_{u}=2$. However, requiring even more regularity for $\boldsymbol{u}$ causes a more unstable pressure solutions; compare Figure 5.12. Besides this, the tests show that an increased regularity in the pressure variable for $r_{p}>1$ can lead to stable and adequate approximations too, even if $r_{u}=r_{p}$ as shown in Figure 5.12. This indicates that a more detailed study of the stability issue is reasonable to obtain a more mathematical criterion of which degrees and regularities give stable numerical results.

The conclusion of this section is as follows: For the space-time method, pressure oscillations but also locking may be present, mainly in the case of small permeability and large Lamé parameters. Mixed polynomial degrees stabilize the numerical solution, but nevertheless, discontinuous data lead, independent of the polynomial degrees, to local overshoots.

6. Concluding remarks. We have introduced and analyzed a novel isogeometric method for the Biot two-field system. It is based on a space-time discretization and allows us to use the spline machinery to achieve arbitrary high convergence order, given sufficient regularity of the 




(a) Terzaghi test problem $\left(c_{0}=k=10^{-7}\right)$.



(b) Pressure for low-permeable layer.

FIG. 5.12. The plots demonstrate the influence of the smoothness of the spatial test function. In the left plot we see the results for the Terzaghi stability test problem from above for the three cases $r_{u}=2, r_{p}=1$, with $C^{1}$-regular displacement test functions (blue curve), $r_{u}=3, r_{p}=1$ with $C^{2}$-regular displacement (black dotted curve), and $r_{u}=2, r_{p}=2$ with $C^{1}$-regular pressure and continuous displacements. On the right, we present the low-permeable layer example for the same degree and regularity parameters. We can observe a more unstable pressure solution if we require smooth displacements $\left(C^{2}\right.$-regularity), whereas an increase of the pressure regularity leads to a stabilized pressure solution, although the same polynomial degrees $r_{u}=r_{p}=2$ are chosen.

exact solution. By several numerical examples we have validated the theory and demonstrated the applicability, even for a 3D geometry. Moreover, the well-known problem of pressure oscillations has been addressed.

In our view, there are two major issues which should be treated in future work. On the one hand, although mixed methods lead to substantial improvements, a closer look at the pressure instability from a theoretical point of view might be advisable in the context of the space-time approach. On the other hand it is desirable to consider the linear algebra for the resulting large-scale linear system in detail. Eventually, this may make the space-time approach also quite competitive with respect to computing times, especially, on parallel computers.

\section{REFERENCES}

[1] G. Alessandrini, A. Morassi, And E. Rosset, The linear constraints in Poincaré and Korn type inequalities, Forum Math., 20 (2008), pp. 557-569.

[2] J. B. Altmann, Poroelastic Effects in Reservoir Modelling, PhD. Thesis, Dept. of Physics, Karlsruher Institut für Technologie (KIT), Karlsruhe, 2010.

[3] M. A. Augustin, A Method of Fundamental Solutions in Poroelasticity to Model the Stress Field in Geothermal Reservoirs, Birkhäuser, Cham, 2015.

[4] M. BAUSE, F. A. RADU, AND U. KÖCHER, Space-time finite element approximation of the Biot poroelasticity system with iterative coupling, Comput. Methods Appl. Mech. Engrg., 320 (2017), pp. 745-768.

[5] Y. BAZilevs, L. Beirão da Veiga, J. A. Cottrell, T. J. R. Hughes, And G. SAngalli, Isogeometric analysis: approximation, stability and error estimates for h-refined meshes, Math. Models Methods Appl. Sci., 16 (2006), pp. 1031-1090.

[6] L. Beirão Da Veiga, D. Cho, And G. SANGAlli, Anisotropic NURBS approximation in isogeometric analysis, Comput. Methods Appl. Mech. Engrg., 209/212 (2012), pp. 1-11.

[7] Y. W. Bekele, E. Fonn, T. Kvamsdal, A. M. KVArving, and S. Nordal, On mixed isogeometric analysis of poroelasticity, Preprint on arXiv, 2017. https: / / arxiv . org/abs/1706.01275

[8] L. Berger, A Low Order Finite Element Method for Poroelasticity with Applications to Lung Modelling, PhD. Thesis, University of Oxford, Oxford, 2015. https: / / arxiv.org/abs/1609.06892 
[9] L. BERGER, R. Bordas, D. KAY, AND S. TAVENER, A stabilized finite element method for finite-strain three-field poroelasticity, Comput. Mech., 60 (2017), pp. 51-68.

[10] M. A. Віот, Le problem de la consolidation des matieres argileuses sous une charge, Ann. Soc. Sci. Bruxelles, B55 (1935), pp. 110-113.

[11] _ General theory of three-dimensional consolidation, J. Appl. Phys., 12 (1941), pp. 155-164.

[12] - Theory of elasticity and consolidation for a porous anisotropic solid, J. Appl. Phys., 26 (1955), pp. 182-185.

[13] D. BRAESS, Finite Elemente, 4th ed., Springer, Berlin, 2007.

[14] A. Buffa AND G. SANGALLI, eds., Isogeometric Analysis: A New Paradigm in the Numerical Approximation of PDEs, Lecture Notes in Mathematics, vol. 2161, Springer, Cham, 2016.

[15] M. CocCO AND J. R. RICE, Pore pressure and poroelasticity effects in coulomb stress analysis of earthquake interactions, J. Geophys. Res.: Solid Earth, 107 (2002), pp. ESE 2-1-ESE 2-17.

[16] C. DE FALCO, A. REALI, AND R. VÁZQUEZ, GeoPDEs: a research tool for isogeometric analysis of PDEs, Adv. Eng. Softw., 42 (2011), pp. 1020-1034.

[17] K. Deng, Y. LiU, And R. M. HarRington, Poroelastic stress triggering of the December 2013 Crooked Lake, Alberta, induced seismicity sequence, Geophys. Res. Lett., 43 (2016), pp. 8482-8491.

[18] G. FU, A high-order HDG method for the Biot's consolidation model, Comput. Math. Appl., 77 (2019), pp. 237-252.

[19] G. GEymonat, Trace theorems for Sobolev spaces on Lipschitz domains. Necessary conditions, Ann. Math. Blaise Pascal, 14 (2007), pp. 187-197.

[20] C. GRÄSER, A note on Poincaré- and Friedrichs-type inequalities, Preprint on arXiv, 2015. https://arxiv.org/abs/1512.02842

[21] J. B. Haga, H. Osnes, AND H. P. LANGTANGen, On the causes of pressure oscillations in low-permeable and low-compressible porous media, Int. J. Numer. Anal. Meth. Geomech., 36 (2011), pp. 1507-1522.

[22] G. HARPER, J. LiU, S. TAVENER, AND Z. WANG, A two-field finite element solver for poroelasticity on quadrilateral meshes, in Computational Science-ICCS 2018. Part III, Y. Shi, H. Fu, Y. Tian, V. Krzhizhanovskaya, M. H. Lees, J. Dongarra, and P. M. A. Sloot, eds., vol. 10862 of Lecture Notes in Comput. Sci., Springer, Cham, 2018, pp. 76-88.

[23] T. J. R. Hughes, J. A. CotTRELl, AND Y. BAZILEvs, Isogeometric analysis: CAD, finite elements, NURBS, exact geometry and mesh refinement, Comput. Methods Appl. Mech. Engrg., 194 (2005), pp. 4135-4195.

[24] F. IRZAL, J. J. REMmers, C. V. Verhoosel, AND R. DE BORST, Isogeometric finite element analysis of poroelasticity, Int. J. Numer. Anal. Meth. Geomech., 37 (2013), pp. 1891-1907.

[25] T. Kraft, P. M. Mai, S. Wiemer, N. Deichmann, J. Ripperger, P. Kästli, C. Bachmann, D. Fäh, J. WÖSSNER, AND D. GIARDINI, Enhanced geothermal systems: mitigating risk in urban areas, Eos Trans. AGU, 90 (2009), pp. 273-274.

[26] U. LANGer, S. E. Moore, AND M. NeUmÜLler, Space-time isogeometric analysis of parabolic evolution problems, Comput. Methods Appl. Mech. Engrg., 306 (2016), pp. 342-363.

[27] J. J. LEE, Robust three-field finite element methods for Biot's consolidation model in poroelasticity, BIT, 58 (2018), pp. 347-372.

[28] A. Malandrino and E. Moeendarbary, Poroelasticity of living tissues, in Encyclopedia of Biomedical Engineering, R. Narayan, ed., Elsevier, Amsterdam, 2019, pp. 238-245.

[29] MATHWorks, Matlab Version 9.6 (R2019a), Natick, 2019.

[30] R. OYARZÚA AND R. RUIZ-BAIER, Locking-free finite element methods for poroelasticity, SIAM J. Numer. Anal., 54 (2016), pp. 2951-2973.

[31] P. J. PHILLIPS, Finite Element Methods in Linear Poroelasticity: Theoretical and Computational Results, PhD. Thesis, University of Texas, Austin, ProQuest LLC, Ann Arbor, 2005.

[32] P. J. Phillips AND M. F. WheEler, A coupling of mixed and continuous Galerkin finite element methods for poroelasticity. I. The continuous in time case, Comput. Geosci., 11 (2007), pp. 131-144.

[33] _ - A coupling of mixed and continuous Galerkin finite element methods for poroelasticity. II. The discrete-in-time case, Comput. Geosci., 11 (2007), pp. 145-158.

[34] R. E. Showalter, Diffusion in poro-elastic media, J. Math. Anal. Appl., 251 (2000), pp. 310-340.

[35] B. Simeon, Computational Flexible Multibody Dynamics, Springer, Heidelberg, 2013.

[36] K. TerZAGHI, Erdbaumechanik auf Bodenphysikalischer Grundlage, F. Deuticke, Leipzig, 1925.

[37] K. VAN THIENEN-VISSER AND J. BREUNESE, Induced seismicity of the Groningen gas field: history and recent developments, The Leading Edge, 34 (2015), pp. 664-671.

[38] J. C. Vardakis, L. Guo, T. W. Peach, T. Lassila, M. Mitolo, D. Chou, Z. A. Taylor, S. Varma, A. VENNERI, A. F. FRANGI, AND Y. VENTIKos, Fluid-structure interaction for highly complex, statistically defined, biological media: homogenisation and a 3D multi-compartmental poroelastic model for brain biomechanics, J. Fluid. Struct., 91 (2019), Art. 102641, 16 pages.

[39] R. VÁZQUEZ, A new design for the implementation of isogeometric analysis in Octave and Matlab: GeoPDEs 3.0, Comput. Math. Appl., 72 (2016), pp. 523-554. 
[40] S.-Y. YI, Convergence analysis of a new mixed finite element method for Biot's consolidation model, Numer. Methods Partial Differential Equations, 30 (2014), pp. 1189-1210.

[41] G. Zhai, M. Shirzaei, M. Manga, And X. Chen, Pore-pressure diffusion, enhanced by poroelastic stresses, controls induced seismicity in Oklahoma, Proc. Nat. Acad. Sci. U.S.A., 116 (2019), pp. 16228 16233.

[42] Y. ZHENG, R. BURRIDGE, AND D. BURNS, Reservoir simulation with the finite element method using Biot poroelastic approach, Tech. Rep., Earth Resources Laboratory, Massachusetts Institute of Technology, Cambridge, 2003. 